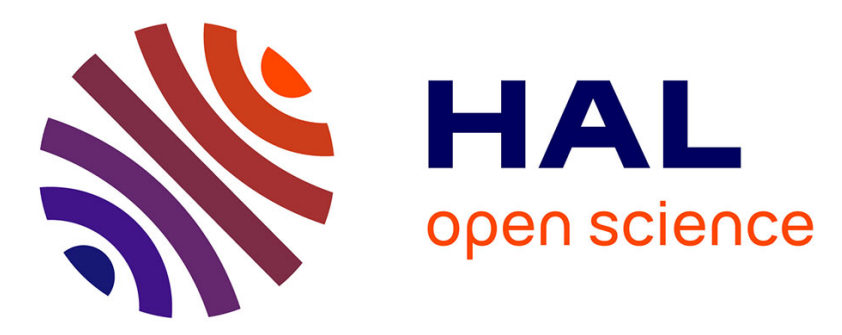

\title{
Absolute instabilities in eccentric Taylor-Couette-Poiseuille flow
}

Colin Leclercq, Benoît Pier, Julian F. Scott

\section{To cite this version:}

Colin Leclercq, Benoît Pier, Julian F. Scott. Absolute instabilities in eccentric Taylor-CouettePoiseuille flow. Journal of Fluid Mechanics, 2014, 741, pp.543-566. 10.1017/jfm.2013.646 . hal00948589

\section{HAL Id: hal-00948589 \\ https://hal.science/hal-00948589}

Submitted on 18 Feb 2014

HAL is a multi-disciplinary open access archive for the deposit and dissemination of scientific research documents, whether they are published or not. The documents may come from teaching and research institutions in France or abroad, or from public or private research centers.
L'archive ouverte pluridisciplinaire HAL, est destinée au dépôt et à la diffusion de documents scientifiques de niveau recherche, publiés ou non, émanant des établissements d'enseignement et de recherche français ou étrangers, des laboratoires publics ou privés. 


\title{
Absolute instabilities in eccentric Taylor-Couette-Poiseuille flow
}

\author{
Colin Leclercq †, Benoît Pier and Julian F. Scott \\ Laboratoire de mécanique des fluides et d'acoustique, \\ École centrale de Lyon-CNRS-Université Claude-Bernard Lyon 1-INSA Lyon, \\ 36 avenue Guy-de-Collongue, 69134 Écully cedex, France
}

(Received 18 July 2013; revised 13 November 2013; accepted 30 November 2013)

The effect of eccentricity on absolute instabilities (AI) in the Taylor-Couette system with pressure-driven axial flow and fixed outer cylinder is investigated. Five modes of instability are considered, characterised by a pseudo-angular order $m$, with here $|m| \leqslant 2$. These modes correspond to toroidal $(m=0)$ and helical structures $(m \neq 0)$ deformed by the eccentricity. Throughout the parameter range, the mode with the largest absolute growth rate is always the Taylor-like vortex flow corresponding to $m=0$. Axial advection, characterised by a Reynolds number $R e_{z}$, carries perturbations downstream, and has a strong stabilising effect on AI. On the other hand, the effect of the eccentricity $e$ is complex: increasing $e$ generally delays AI, except for a range of moderate eccentricites $0.3 \lesssim e \lesssim 0.6$, where it favours AI for large enough $R e_{z}$. This striking behaviour is in contrast with temporal instability, always inhibited by eccentricity, and where left-handed helical modes of increasing $|m|$ dominate for larger $R e_{z}$. The instability mechanism of $\mathrm{AI}$ is clearly centrifugal, even for the larger values of $R e_{z}$ considered, as indicated by an energy analysis. For large enough $R e_{z}$, critical modes localise in the wide gap for low $e$, but their energy distribution is shifted towards the diverging section of the annulus for moderate $e$. For highly eccentric geometries, AI are controlled by the minimal annular clearance, and the critical modes are confined in the vicinity of the inner cylinder. Untangling the AI properties of each $m$ requires consideration of multiple pinch points.

Key words:

\section{Introduction}

The flow between rotating cylinders has attracted attention since the end of the $19^{\text {th }}$ century, starting with the experiments of Couette $(1888 b, a)$ and Mallock (1888), and the landmark work by Taylor (1923), who first predicted theoretically the threshold for centrifugal instability. Taylor characterised centrifugal effects using a non-dimensional number appropriate in the limit of small clearance, $d=(b-a) \ll a$, with $a$ and $b$ the inner and outer cylinder radii (see figure 1). In this paper, a wide gap geometry with radii ratio $\eta=a / b=0.5$ will be considered, and centrifugal effects will be conveniently measured by an azimuthal Reynolds number $R_{\Omega}=a \Omega d / \nu$, with $\Omega$ the inner cylinder rotation rate and $\nu$ the kinematic viscosity.

Adding axial flow to this system, one obtains a simple prototype for the study of pattern formation in real open flows. The effect of axial advection can also be measured by

$†$ Email address for correspondence: colin.leclercq@ec-lyon.fr 


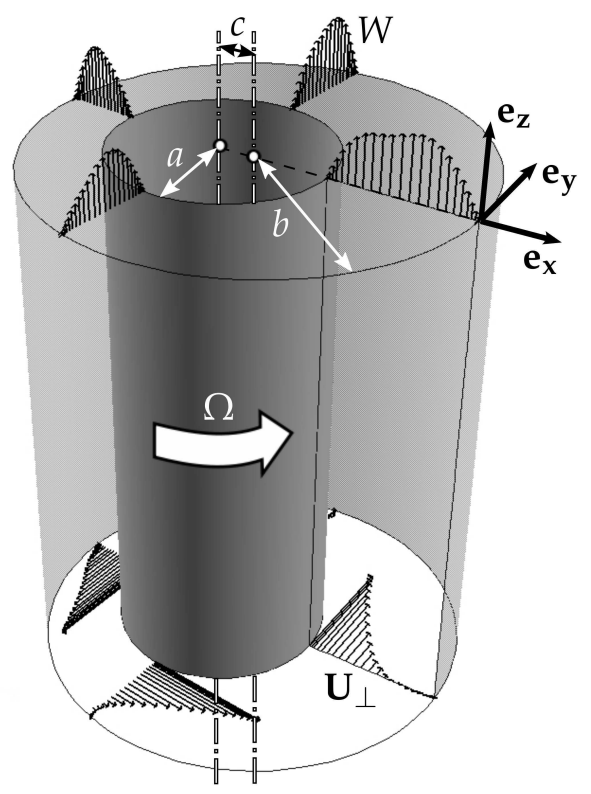

FiguRE 1. Eccentric annulus of radius ratio $\eta=a / b=0.5$ and basic flow $\mathbf{U}=\mathbf{U}_{\perp}+W \mathbf{e}_{\mathbf{z}}$.

a Reynolds number $R e_{z}=\bar{W} d / \nu$, based on the mean axial velocity $\bar{W}$. First theoretical predictions of the absolute instability (AI) threshold were obtained by Tsameret \& Steinberg (1991a) with a criterion based on a one-dimensional Ginzburg-Landau equation (with coefficients determined by two-dimensional numerical simulations), and then by Babcock et al. (1991, 1992), using the full set of hydrodynamic equations and a saddlepoint criterion (Briggs 1964; Bers 1983) that will be discussed in $§ 2.3$. They showed that upon crossing the AI threshold, periodic self-sustained vortices appear, in contrast with the irregular patterns emerging from noise amplification in the convectively unstable régime. In these papers and subsequent work (Tsameret \& Steinberg 1991b; Babcock et al. 1992; Lücke \& Recktenwald 1993; Swift et al. 1994; Babcock et al. 1994; Tsameret \& Steinberg 1994), effort was dedicated to identifying the noise sources (inlet noise versus thermal noise) which sustain the convective instability (CI). These studies were restricted to small axial Reynolds numbers $R e_{z}$, typically below 4 , and it was found that the most unstable (fastest growing) perturbations were in the form of propagating Taylor vortices. For higher values of axial advection, Takeuchi \& Jankowski (1981) and Ng \& Turner (1982) had previously shown numerically (and also experimentally for the former reference) that critical modes consisted in propagating helical vortices, with helicity opposite that of the basic flow, and with azimuthal order $m$ increasing with $R e_{z}$. However, the concept of AI was not widespread in fluid mechanics back then, and these studies were restricted to CI. Theoretical prediction of AI of helical modes was investigated only recently (Hoffmann 2004, Altmeyer 2011). In these papers, the authors studied the effect of axial through-flow on the spatio-temporal properties of toroidal and helical wavepackets with angular orders $|m| \leqslant 2$, for $R e_{z} \leqslant 20$. For $|m| \leqslant 1$, it was shown that critical azimuthal Reynolds numbers $R e_{\Omega}$ are higher for AI than for CI, and that the difference between the two thresholds increases with $R e_{z}$. For $|m|=2$ and a stationary outer cylinder, AI was found to occur in a closed region of the $R e_{z}-R e_{\Omega}$ plane, considering only the saddle point originating at the critical conditions for CI with $R e_{z}=0$ (detailed in $\S 2.4$ ). However, the authors mentioned other saddle points expected to destabilise these modes 
in other regions of parameter space and which will be taken into account in the present article. More recent work on AI in the Taylor-Couette-Poiseuille flow concerned the effect of radial flow at the inner cylinder, representative of filtration devices (Martinand et al. 2009). In this analysis, it was shown that axisymmetric modes become absolutely unstable for inward radial flow, while helical modes with helicity identical to that of the basic flow dominate at high enough $R e_{z}$, for outward radial flow.

When the two cylinder axes do not coincide, axisymmetry is broken and the stability properties of the flow are modified. Eccentricity is generally measured by the nondimensional distance between the two cylinders $e=c / d$ (see figure 1). Adding eccentricity to the Taylor-Couette flow with axial advection, one obtains a basic model for annular mud flows in oil-well drilling, or lubrication flows present in high-speed journal bearings. In the first case, mud is injected in a rotating drillstring, and flows back to the surface through the annular domain between the drillstring and the rock face, with several engineering functions: carry the rock cuttings out, lubricate, prevent inflow of formation gases and wellbore collapse, etc. (Escudier et al. 2002; Guo \& Liu 2011). For deep wells, the drillstring inevitably bends along its axis, on a typical length scale much larger than the well diameter. As a result, a parallel-flow assumption is reasonable, and the flow can be locally described as a Taylor-Couette-Poiseuille flow between eccentric cylinders. In high-speed turbomachinery, a similar configuration is found: oil is contained in eccentric journal bearings for lubrication purposes, and a pressure gradient is imposed along the shaft to evacuate damaging impurities (Sep 2008). Aside from its fundamental interest, these industrial applications motivate the present analysis. In both applications, transition to complex hydrodynamic régimes would result in increased frictional losses, detrimental to the system efficiency. If the basic flow advection is weak compared to the rotation rate, hydrodynamic resonance may occur and the entire flow would bifurcate to an undesired self-sustained oscillatory state. This specific behaviour, called absolute instability, is particularly 'dangerous', because it does not require a permanent forcing: once the instability is triggered, it will propagate in both the downstream and upstream directions, and amplify using energy from the basic flow. On the other hand, convective instabilities correspond to wavepackets propagating only in the downstream direction: in the absence of forcing, the system eventually relaxes to its initial state at any fixed location, after perturbations have been 'blown away' from the source. The most temporally amplified perturbations are given by a classical temporal stability analysis, and such a study was recently carried out for this flow (Leclercq et al. 2013). It was shown that the physics is essentially similar to the axisymmetric case (Takeuchi \& Jankowski 1981; Ng \& Turner 1982), with propagating toroidal vortices replaced by helical structures of increasing azimuthal complexity as $R e_{z}$ is increased. Eccentricity deforms the critical modes, but does not introduce new instabilities to the problem. The effect of eccentricity is stabilising for all values of $R e_{z}$, and this result is interpreted as a consequence of the reduction of centrifugal effects in the basic flow. Indeed, as eccentricity increases, the azimuthal flow rate decreases for a fixed inner cylinder rotation rate, resulting in weaker driving of the instability. This is a consequence of the appearance of a recirculation region in the wide gap which does not contribute to the net azimuthal flow rate. Outside this zone, in the vicinity of the inner cylinder, the flow resembles an axisymmetric Taylor-Couette flow with clearance scaling with the inner gap $d(1-e)$. The reduction of the 'effective' clearance ratio $\delta=d / a$ with eccentricity, or increase in 'effective' radius ratio $\eta$, also explains stabilisation (see DiPrima (1960) for the effect of $\eta$ on the Taylor-Couette flow). To date, it is, to the authors' knowledge, the only available theoretical study of eccentric Taylor-Couette-Poiseuille flow. The only known series of experiments were performed by Coney \& Mobbs (1969-70); Coney (1971); Younes (1972); Younes et al. (1972); Mobbs \& 
Younes (1974); Coney \& Atkinson (1978) and show good agreement with our a posteriori predictions, despite small discrepancies attributed to finite-length effects. For a brief review of other theoretical and experimental results on eccentric Taylor-Couette flow on the one hand, and axisymmetric Taylor-Couette-Poiseuille flow on the other hand, we refer to Leclercq et al. (2013).

The present paper extends this previous linear stability analysis by considering the case of AI. In $\S 2$, the linear stability framework is presented. The governing equations and numerical methods are briefly described, and the main properties of the basic flow and normal modes are recalled. In $\S 2.3$, the methods used to investigate AI, based on the Briggs (1964)-Bers (1983) pinching criterion, are described. In $\S 3$, results are presented for the 5 modes of instability with angular orders $|m| \leqslant 2$, which include the fastest growing temporally unstable modes for $R e_{z} \leqslant 50$.

\section{Linear stability framework}

In the following, the geometry will be described using the ratio $0<\eta=a / b<1$ between the inner and outer cylinder radii $a$ and $b$ (see figure 1), and the eccentricity $0 \leqslant e=c /(b-a)<1$, based on the distance $c$ between centers, divided by the clearance $d=b-a$. The gap varies azimuthally between $d(1-e)$ and $d(1+e)$. The radii ratio will be fixed at the value $\eta=0.5$ throughout this paper. Rotation and axial advection will be quantified using the two Reynolds numbers given in introduction: $R e_{\Omega}=a \Omega d / \nu$ and $R e_{z}=\bar{W} d / \nu$, with $\Omega$ the inner cylinder rotation rate, $\bar{W}$ the basic flow mean axial velocity and $\nu$ the kinematic viscosity.

The velocity $\mathbf{u}$ will be made non-dimensional with the rotation speed $V \equiv a \Omega$. The clearance $d$ will be taken as the reference length scale $L$. Finally, the pressure $p$ will be in units of $P \equiv \rho V^{2}$, with $\rho$ the density of the fluid. All equations and physical quantities will be written in non-dimensional form, using $V, L$ and $P$.

\subsection{Basic flow}

The velocity $\mathbf{u}$ can be decomposed into a component $w$ parallel to the axis $\mathbf{e}_{z}$, and a component $\mathbf{u}_{\perp}=\mathbf{u}-w \mathbf{e}_{z}$ in a plane perpendicular to the axis. The axial flow is driven by a pressure gradient $G$ in the $z$-direction. Denoting the in-plane pressure gradient as $\nabla_{\perp} p=\nabla p-G \mathbf{e}_{z}$, the incompressible Navier-Stokes equations read:

$$
\left.\begin{array}{rl}
\left(\partial_{t}+\mathbf{u} \cdot \boldsymbol{\nabla}\right)\left[\begin{array}{c}
\mathbf{u}_{\perp} \\
w
\end{array}\right] & =-\left[\begin{array}{c}
\nabla_{\perp} p \\
G
\end{array}\right]+\operatorname{Re}_{\Omega}^{-1} \nabla^{2}\left[\begin{array}{c}
\mathbf{u}_{\perp} \\
w
\end{array}\right], \\
\boldsymbol{\nabla} \cdot \mathbf{u} & =0,
\end{array}\right\}
$$

with impermeability and no-slip boundary conditions on the fixed outer cylinder and on the inner cylinder, whose rotational velocity is 1 . In-plane and axial derivatives can be separated, using convenient notations: $\mathbf{u}_{\perp} \cdot \nabla_{\perp} \equiv \mathbf{u} \cdot \boldsymbol{\nabla}-w \partial_{z}, \nabla_{\perp}^{2} \equiv \nabla^{2}-\partial_{z z}^{2}$ and $\boldsymbol{\nabla}_{\perp} \cdot \mathbf{u}_{\perp} \equiv \boldsymbol{\nabla} \cdot \mathbf{u}-\partial_{z} w$. Assuming an axially invariant flow, the problem is two-dimensional and $\mathbf{u}_{\perp}$ becomes independent of $w$ :

$$
\left.\begin{array}{rl}
\partial_{t} \mathbf{u}_{\perp}+\mathbf{u}_{\perp} \cdot \nabla_{\perp} \mathbf{u}_{\perp} & =-\nabla_{\perp} p+R e_{\Omega}^{-1} \nabla_{\perp}^{2} \mathbf{u}_{\perp}, \\
\nabla_{\perp} \cdot \mathbf{u}_{\perp} & =0 .
\end{array}\right\}
$$

Basic flows $\mathbf{Q} \equiv(\mathbf{U}, P)$, denoted with capital letters, are defined as axially invariant, steady solutions of (2.1). Such solutions are found by integrating forwards in time equation (2.2) until convergence of $\mathbf{U}_{\perp}$ is attained, and then solving for the corresponding 


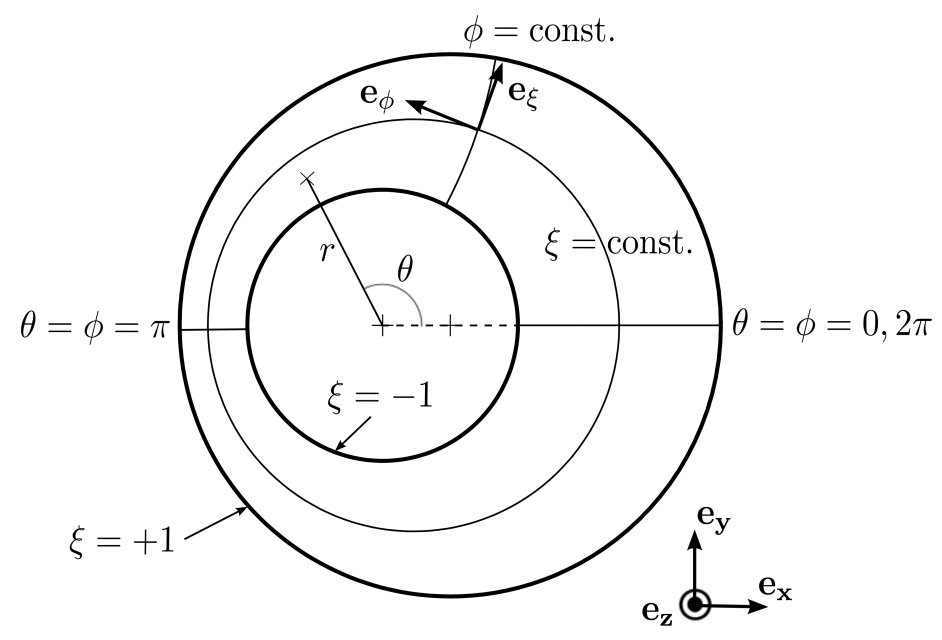

FIgURE 2. Modified bipolar coordinate system $(\xi, \phi)$ fitting the eccentric annular domain. Polar coordinates $(r, \theta)$ are centered on the inner cylinder, with $\theta=\phi=0$ along the line joining the cylinder axes.

axial velocity $W$, given by:

$$
\mathbf{U}_{\perp} \cdot \nabla_{\perp} W=-G+R e_{\Omega}^{-1} \nabla_{\perp}^{2} W .
$$

Equations are expressed using locally orthogonal, body-fitted coordinates $(\xi, \phi)$, with $-1 \leqslant \xi \leqslant 1$ and $0 \leqslant \phi<2 \pi$ the pseudo-radial and pseudo-azimuthal coordinates respectively (see figure 2). In this modified bipolar coordinate system, a Fourier-Chebyshev pseudospectral projection method is implemented, with $N_{\phi}=2 K_{\phi}+1$ Fourier modes, and $N_{\xi}$ Gauss-Lobatto collocation points. For more details on the numerical procedure, the reader is referred to Leclercq et al. (2013).

For an axisymmetric flow, the basic in-plane motion results from diffusion of axial vorticity from the rotating inner cylinder to the fixed outer cylinder. In cylindrical coordinates $(r, \theta)$, it takes the well-known form $\mathbf{U}_{\perp}=(0, A r+B / r)$, with $A$ and $B$ two constants depending on the geometry. For low eccentricities, the result is quite similar, as can be seen in figure 3(1a). However, for higher eccentricities, a low-speed recirculation region forms in the wide gap (figure $3(2 a)$ ). For the relatively high value of $R e_{\Omega}=500$ presented here, small recirculation can already be seen for $e=0.2$, whereas in Leclercq et al. (2013), figure 3, it was not present for $R e_{\Omega}=100$ and appeared around $e \approx 0.3$ for that lower value of $R e_{\Omega}$.

In the axisymmetric case, $W$ is independent of $\mathbf{U}_{\perp}$, and the axial flow is very similar to a parabolic Poiseuille flow, with small corrections due the annular geometry. As eccentricity is increased, $W$ decreases in the small gap, because of viscous effects, and most of the volume flux passes through the wide gap (see figure 3(2b)). Distortion also occurs, due to coupling with $\mathbf{U}_{\perp}$, and the peak velocity is no longer in the symmetry plane. For high rotation rates, the nonlinear interaction term $\mathbf{U}_{\perp} \cdot \nabla_{\perp} W$ can locally dominate the viscous term $R e_{\Omega}^{-1} \nabla_{\perp}^{2} W$, and there is significant transport of $W$ by in-plane components.

\subsection{Normal modes}

Let $\mathbf{q}^{\prime} \equiv \mathbf{q}-\mathbf{Q}$ be three-dimensional perturbations of small amplitude superimposed onto the two-dimensional basic flow, and satisfying the linearized Navier-Stokes equations with no-slip boundary conditions. Because of temporal and axial invariance of the basic 
(1a)

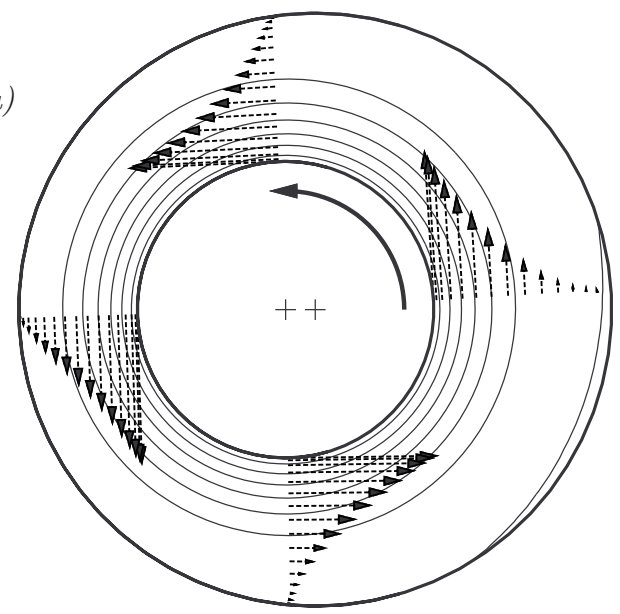

(2a)

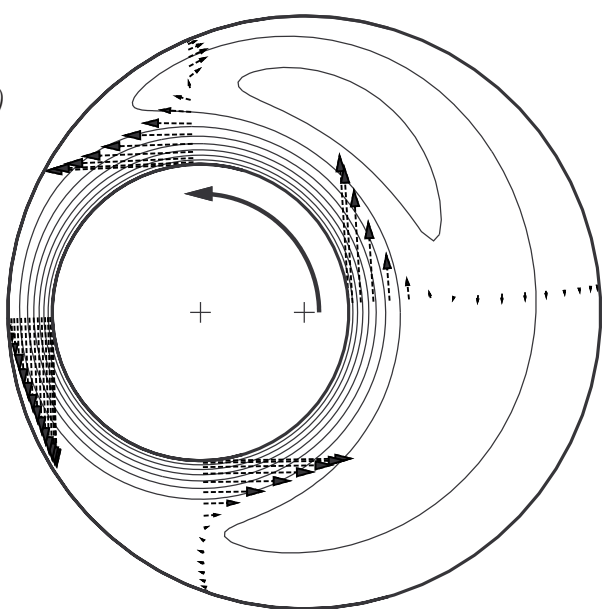

(1b)

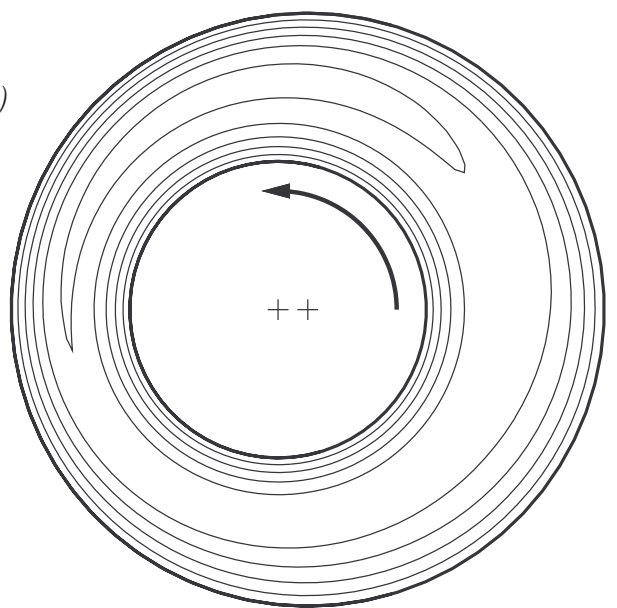

(2b)

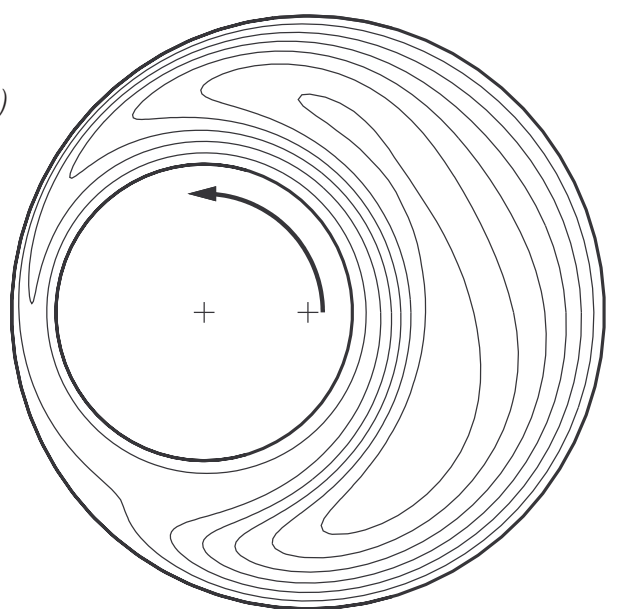

FiguRE 3. Basic flows for $R_{\Omega}=500$ : (1) weak eccentricity $e=0.2$, (2) high eccentricity $e=0.7$. (a) Contours of equispaced in-plane streamfunction with superimposed $\mathbf{U}_{\perp}$ profiles at $\theta=0, \pi / 2, \pi, 3 \pi / 2$ (polar angle with respect to the inner cylinder). (b) Equispaced contours of $W$.

flow, perturbations are sought in the form of normal modes

$$
\mathbf{q}^{\prime}=\tilde{\mathbf{q}}(\xi, \phi) \exp \mathrm{i}(k z-\omega t)+c . c .,
$$

where c.c. denotes the complex conjugate. In a general framework, $k$ is the complex axial wavenumber and $\omega$ is the complex frequency. As usual, $\omega_{\mathrm{r}} \equiv \operatorname{Re}(\omega)$ is the temporal frequency and $\omega_{\mathrm{i}} \equiv \operatorname{Im}(\omega)$ is the temporal growth rate. Equivalently, $k_{\mathrm{r}} \equiv \operatorname{Re}(k)$ is the wavenumber of the mode, and $-k_{\mathrm{i}} \equiv-\operatorname{Im}(k)$ is the spatial growth rate. Injecting the modal form (2.4) into the linearized Navier-Stokes equations with boundary conditions, one obtains a problem of the form $(\mathcal{A}-\mathrm{i} \omega \mathcal{B}) \tilde{\mathbf{q}}=0$, where $(\mathcal{A}, \mathcal{B})$ are two linear operators, with $\mathcal{A}$ depending on $k$. Expressions for $\mathcal{A}$ and $\mathcal{B}$ are given in Leclercq et al. (2013), in the modified bipolar coordinate system. The wavenumbers $k$ and frequencies $\omega$ satisfying this problem for non-zero $\tilde{\mathbf{q}}$ define the dispersion relation $D(k, \omega)=0$. Using the same Fourier-Chebyshev decomposition as for the basic flow, the linear problem is converted into a generalized eigenvalue problem for $\omega$ and $\tilde{\mathbf{q}}$ that can be solved numerically, using LAPACK (www.netlib.org/lapack) or ARPACK++ (Lehoucq et al. 1997) routines. For 


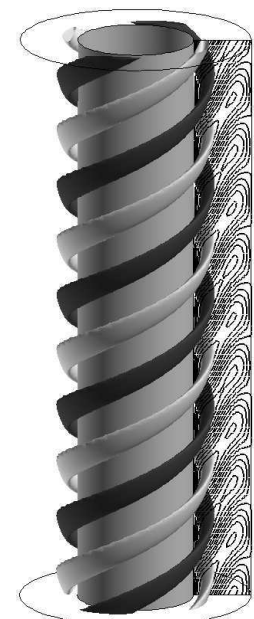

RH2

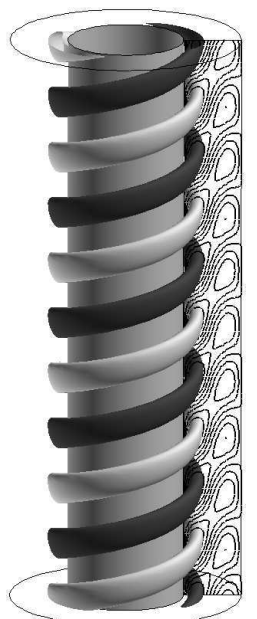

RH1

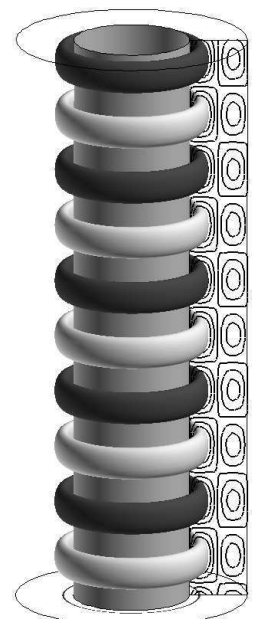

TV

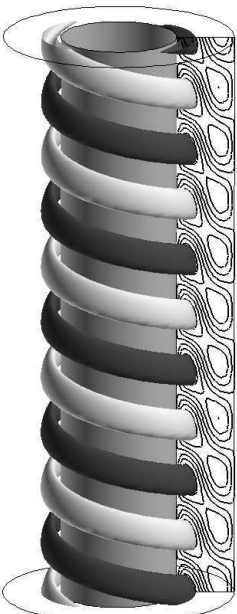

LH1

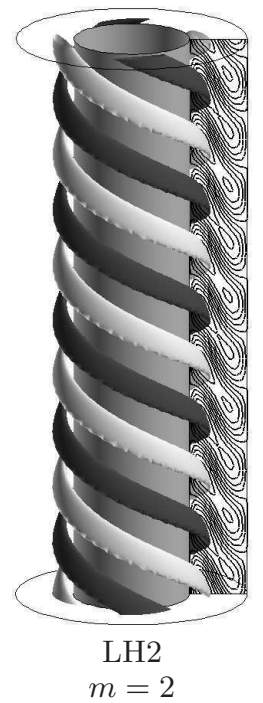

Figure 4. Structure (isovalues of axial velocity) of the five modes of interest $m=-2, \ldots, 2$, from left to right. For this example, $e=0, R e_{\Omega}=68.19, R e_{z}=0$ and $k=3.16$, corresponding to the critical conditions for temporal instability of the axisymmetric Taylor-Couette flow (see DiPrima \& Swinney (1985) for a review).

more information on the numerical procedure, the reader is referred to Leclercq et al. (2013).

In this previous study, a temporal stability analysis was carried out to predict the fastest growing perturbations with $k$ real and $\omega$ complex. It was found that among the large set of temporal modes $\omega(k)$, the most unstable ones were in the form of deformed toroidal vortices for low $R e_{z}$, or complex helical structure for higher $R e_{z}$. The modes were labelled according to a pseudo-azimuthal-integer-wavenumber $m$, or angular order. The labelling was made in accordance to the axisymmetric case, where normal modes can be written as $\mathbf{q}^{\prime}=\tilde{\mathbf{q}}(r) \operatorname{expi}(k z+m \theta-\omega t)$ in polar coordinates $(r, \theta)$ (see figure 2). Restricting attention to positive $k$, because of symmetry arguments to be discussed in the next paragraph, positive values of $m$ (resp. negative) correspond to helical structures winding clockwise (resp. counter-clockwise) around the inner cylinder, and were called left-helical (resp. right-helical), or $\mathrm{LH}|m|$ (resp. $\mathrm{RH}|m|$ ) modes. The case $m=0$ corresponds to the classical toroidal Taylor vortex flow (TV). By following these modes as eccentricity is continuously varied, one obtains the corresponding pseudo-angular-order $m$ for $e \neq 0$. Figure 4 shows the structure of modes $m=-2, \ldots, 2$ for the classical Taylor-Couette flow.

Note that the symmetry $\Pi_{0} \equiv(m, \omega, w) \rightarrow\left(-m,-\omega^{\star},-w\right)$ (with * denoting the complex conjugate) between $\mathrm{RH}$ and $\mathrm{LH}$ in figure 4 is broken when axial flow is added, or when $k$ is complex. Indeed, by taking the complex conjugate of the axisymmetric modal form, the general symmetry $\Pi_{1} \equiv(k, m, \omega) \rightarrow\left(-k^{\star},-m,-\omega^{\star}\right)$ appears, also valid for $e \neq 0$. By considering the mirror image of the system $(z \rightarrow-z)$, one obtains another symmetry: $\Pi_{2} \equiv\left(R e_{z}, k, w\right) \rightarrow\left(-R e_{z},-k,-w\right)$. Combining $\Pi_{1}$ and $\Pi_{2}$, one gets

$$
\Pi_{3} \equiv\left(R e_{z}, k, m, \omega, w\right) \rightarrow\left(-R e_{z}, k^{\star},-m,-\omega^{\star},-w\right) .
$$

Setting $R e_{z}$ to zero and $k$ real in $\Pi_{3}$, one recovers $\Pi_{0}$. In the general case, because of $\Pi_{3}$, one can choose to study only $m \geqslant 0$, or only $R e_{z} \geqslant 0$, without loss of generality. $\Pi_{1}$ also indicates that it is possible to restrict computations to $k_{\mathrm{r}} \geqslant 0$. 


\subsection{Absolute instability threshold}

Absolute instability occurs when the impulse response wavepacket is temporally growing at any fixed axial position $z$. The asymptotic dynamics of the wavepacket is dominated by the normal mode which satisfies the pinching criterion of Briggs (1964)-Bers (1983) and has the largest temporal growth rate. This mode has zero group velocity $\partial \omega / \partial k\left(k_{0}\right)=0$ for the complex absolute wavenumber $k_{0}$. This condition indicates the presence of a saddle point of $\omega(k)$ at $k_{0}$. This saddle point is associated to a branch-point singularity at the complex absolute frequency $\omega_{0}=\omega\left(k_{0}\right)$. Additionally, the saddle point must comply with causality and result from the collision between downstream- and upstream-propagating spatial branches, respectively denoted $k^{+}(\omega)$ and $k^{-}(\omega)$. The flow is absolutely unstable if and only if the most unstable pinch point has positive absolute growth rate $\omega_{0, \mathrm{i}}$. Otherwise, the flow is either stable or convectively unstable. In the latter case, the wavepacket grows while being advected away from the impulse location so the system eventually relaxes to its initial state at any axial position. For a comprehensive review of these concepts, the reader is referred to Huerre \& Monkewitz (1990); Huerre (2000); Chomaz (2005).

The border of the absolutely unstable domain is determined by following all the neutrally stable saddle points in parameter space. This is done by performing NewtonRaphson iterations at each point, varying simultaneously $k_{\mathrm{r}}$, $k_{\mathrm{i}}$ and $R e_{\Omega}$ until $\left|\omega_{\mathrm{i}}\right|$, $|\operatorname{Re}(\partial \omega / \partial k)|$ and $|\operatorname{Im}(\partial \omega / \partial k)|$ are all below $10^{-6}$. Estimated values for the independent variables are obtained by linear extrapolation with respect to the parameter being varied, e.g. $e, R e_{\Omega}$ or $R e_{z}$. For $|m|=2$, critical curves display folds, and it is necessary to implement a continuation scheme based on an arclength variable (Keller 1977).

However, not all saddle points are valid and only the ones satisfying the pinching criterion are relevant. In order to discard invalid saddle points, extensive tests are carried out, where the two spatial branches $k(\omega)$ coalescing at $k_{0}$ are tracked numerically as $\omega_{\mathrm{i}}$ is increased from $\omega_{0}$. The saddle point is a genuine pinch only when the spatial branches separate into the upper and lower half $k$-planes for large enough $\omega_{i}$. Indeed, causality demands that this be true for $\omega_{\mathrm{i}}>\omega_{\mathrm{i} \text {,max }}$, where $\omega_{\mathrm{i} \text {, max }}$ is the maximum temporal growth rate for real $k$. Spatial branches are obtained by numerically inverting the relation $\omega(k)$ with a Newton-Raphson iteration.

\subsection{Validation}

To validate the numerical procedure, critical curves in the axisymmetric case were computed and compared with literature results. For $m=0$ and 1, Pinter et al. (2003) provide the coefficients of fourth-order polynomials $f_{m}$ fitting their data in the range $-20 \leqslant R e_{z} \leqslant 20$, with step $\delta R e_{z}=1$. The same procedure was applied here, and our calculated values $\tilde{g}_{m}$ at the same points were fitted by polynomials $g_{m}$. To compare our results, the residual $\sum_{-20}^{20}\left|f_{m}\left(R e_{z}\right)-g_{m}\left(R e_{z}\right)\right|^{2}$ between the two fits was divided by the residual $\sum_{-20}^{20}\left|g_{m}\left(R e_{z}\right)-\tilde{g}_{m}\left(R e_{z}\right)\right|^{2}$ between our fit and our calculated values. For $m=0$ and 1 , this ratio is respectively $1.16 \times 10^{-2}$ and $1.10 \times 10^{-2}$, showing agreement with the authors' calculations. For $m=2$, only graphical data was available, and figure 5 shows excellent agreement with the 'island' of instability found by Altmeyer et al. (2011) in $R e_{\Omega}-R e_{z}$ plane. In their analysis, the authors considered only the saddle points originating at the real critical wavenumber $k_{c}$ of temporal instability with $R e_{z}=0$, as will be explained now. For $R e_{\Omega}$ above the temporal instability threshold $R e_{\Omega, c}$, the medium is unstable, and the growing part of the wavepacket is bounded by two spatio-temporal rays referred to as leading and trailing fronts, respectively $z / t=V^{+}$ and $z / t=V^{-}$with $V^{-}<V^{+}$. The fronts are defined by the conditions $\partial \omega / \partial k\left(k_{*}^{ \pm}\right)=V^{ \pm}$ 


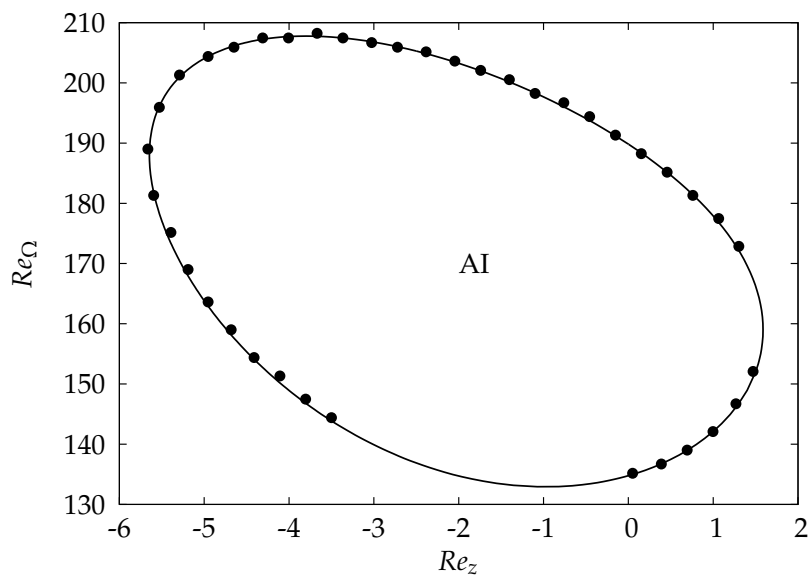

FIgURE 5. 'Island' of AI in the axisymmetric case, for mode LH2. Line: present calculation; dots: figure 8(b) in Altmeyer et al. (2011).

\begin{tabular}{c||ccc|cc|cc|cc|cc}
$m$ & \multicolumn{2}{c||}{0} & \multicolumn{2}{c|}{1} & -1 & \multicolumn{2}{|c|}{2} & \multicolumn{2}{c}{-2} \\
\hline$e$ & 0.3 & 0.7 & 0.3 & 0.7 & 0.3 & 0.7 & 0.45 & 0.7 & 0.45 & 0.7 \\
\hline $16 \times 8$ & 402.59 & 283.22 & 475.66 & 285.22 & 431.88 & 300.21 & 465.54 & 305.73 & 472.77 & 347.83 \\
$16 \times 16$ & 402.59 & 283.32 & 475.66 & 285.70 & 431.88 & 300.22 & 465.54 & 306.79 & 472.77 & 347.90 \\
$32 \times 32$ & 402.59 & 283.33 & 475.65 & 285.71 & 431.88 & 300.29 & 465.52 & 306.80 & 472.46 & 348.34
\end{tabular}

TABLE 1. Critical azimuthal Reynolds number $R e_{\Omega}$ for $R e_{z}=50$ and different resolutions $N_{\xi} \times K_{\phi}$.

and $\omega_{\mathrm{i}}\left(k_{*}^{ \pm}\right)-V^{ \pm} k_{*, \mathrm{i}}^{ \pm}=0$ (see Huerre (2000) for more details). At critical conditions for temporal instability, $R e_{\Omega}=R e_{\Omega, c}$ and $k=k_{c}$, the constraint $\partial \omega_{\mathrm{i}} / \partial k=0$ for $k$ real defines two degenerate fronts propagating at the group velocity $V_{\max }$ of the most rapidly amplified temporal mode: $V^{ \pm}=V_{\max }=\partial \omega / \partial k\left(k_{c}\right)$. For $R e_{\Omega}$ just above $R e_{\Omega, c}, V^{+} \neq V^{-}$ so the fronts are properly defined and the now complex wavenumbers $k_{*}^{ \pm}$are close to $k_{c}$ so $V^{ \pm} \approx V_{\max }$. In general, $V_{\max }>0$, so the wavepacket is advected downstream and the flow is only convectively unstable. But when $R_{\Omega}$ is further increased, one front may eventually change propagation direction, which translates into the saddle-point condition $\partial \omega / \partial k=0$ defining the AI threshold. In Altmeyer et al. (2011), only the two fronts bounding the convectively unstable wavepacket at $R e_{\Omega}$ slightly above $R e_{\Omega, c}$ and $R e_{z}=0$ were considered. These specific fronts were followed as $R e_{\Omega}$ and $R e_{z}$ were varied, and the AI boundary in figure 5 corresponds to points where one of the fronts was stationary. Saddle points corresponding to other stationary fronts were ignored in figure 5 , even though the authors mentioned the existence of more. Note that validation for $m \geqslant 0$ is sufficient because of the $\Pi_{3}$-symmetry (2.5).

In this study, only values of $|m| \leqslant 2$ will be considered and a small number of Fourier modes $K_{\phi}=8$ is deemed satisfactory, as can be seen in table 1 . A higher number of collocation points $N_{\xi}=16$ is however required for accuracy at large $R e_{\Omega}$.

\section{Results}

A parametric study has been performed within the ranges $e \leqslant 0.7,0 \leqslant R e_{z} \leqslant 60$ and $0 \leqslant R e_{\Omega} \leqslant 500$. Within these bounds, modes $m=0,1,2$ are always the most temporally 
unstable, except for a small range $e \leqslant 0.3,50 \leqslant R e_{z} \leqslant 60$, where $m=3$ has the largest temporal growth rate (Leclercq et al. 2013). It will be assumed that the absolute growth rate of these modes will be higher than that of $|m| \geqslant 3$. However, right helical modes $\mathrm{RH} 1$ and $\mathrm{RH} 2$ will be retained in the analysis, as RH are known to be more absolutely unstable than TV and LH in some cases (e.g. high $R e_{z}$, outward radial flow, $e=0$, cf. Martinand et al. (2009)). Because we are considering both positive and negative $m$, it is unnecessary to consider negative $R e_{z}$, because of the $\Pi_{3}$-symmetry $(2.5)$.

\subsection{Reference saddle point}

The bifurcation to (pseudo-)toroidal vortices without axial flow is a steady one, $\omega_{\mathrm{r}}(m=$ $0)=0$, so CI and AI thresholds, respectively denoted here $R e_{\Omega, c}$ and $R e_{\Omega, c-a}$, coincide in this case. For modes $m \neq 0$, CI occurs through Hopf bifurcations at $R e_{z}=0$, so AI only occurs above a higher threshold: $R e_{\Omega, c-a}>\operatorname{Re} e_{\Omega, c}$. For these modes, $R e_{\Omega, c-a}\left(R e_{z}=0\right)$ is found by locating the saddle point with $k_{0}$ closest to $k_{c}$, the real critical wavenumber of CI. This neutral saddle point corresponds to a stationary front of the impulse response wavepacket for $R e_{\Omega}$ just above $R e_{\Omega, c}$, as explained in detail in section $\S 2.4$. For $m=1,2$, the stationary front is the trailing one: $V_{-}=0, k_{0, \mathrm{i}}<0$. For negative $m, k_{0, \mathrm{i}}>0$ because of the $\Pi_{3}$-symmetry $(2.5)$, and the stationary front is the leading one: $V_{+}=0$. The present paragraph defined the reference saddle point for each $m$, obtained for $R e_{z}=0$ and $e=0$. These saddle points are systematically followed in parameter space to define critical curves of AI. However, as will be seen in the next paragraph, other saddle points are also relevant to the spatio-temporal dynamics and must be considered.

\subsection{Multiplicity of saddle points}

Pinch points corresponding to other stationary fronts can be identified using the geometric method described in $§ 3.2$ of Juniper (2006). The same approach is used here to study the dispersion relation of mode $m=0$ in the presence of strong axial advection, $R e_{z}=60$, for three eccentricities around $e=0.3$. Figure 6 , similar to figure 2 in Juniper (2006), shows isocontours of $\omega_{\mathrm{i}}(k)$ for complex values of $k$. The thick black line indicates a contour in the complex $k$-plane including the steepest descent paths of all genuine pinch points, here $s_{1}$ and $s_{2}\left(s_{3}\right.$ and $s_{4}$ are 'spurious' $k^{-} / k^{-}$saddle points). The impulse response can be obtained at any time using a classical inverse Laplace transform formula (see Huerre (2000) for instance), which is easily evaluated at large time using this integration contour. The asymptotic response is indeed dominated by the pinch point of largest temporal growth rate, which here is $s_{2}$. With this geometrical approach, genuine pinch points and invalid saddle points are easily identified.

If the eccentricity is varied by a small amount, the nature of the saddle points may change: valid saddle points may become invalid and vice versa. This is illustrated in figure 7: in case (b), $s_{1}$ and $s_{2}$ are the two pinch points, but in case (a), only $s_{1}$ is a pinch, and in case (c), $s_{1}, s_{2}$ and $s_{3}$ are all three valid. Since the growth rate of each saddle point also varies with the control parameters, the dominant pinch may either be $s_{1}, s_{2}$ or $s_{3}$, and a careful analysis is required when parameters are varied.

Maps similar to figure 6 are drawn for each value of $m$, and a large number of saddle points are identified each time. Saddle points with growth rate close to the referencesaddle-point's are systematically followed in parameter space. Over the whole range of parameters, three different 'pinch points' are found to be relevant for TV, two for LH1, LH2 and RH2, but surprisingly, just one for RH1, despite numerous candidate saddle points. 


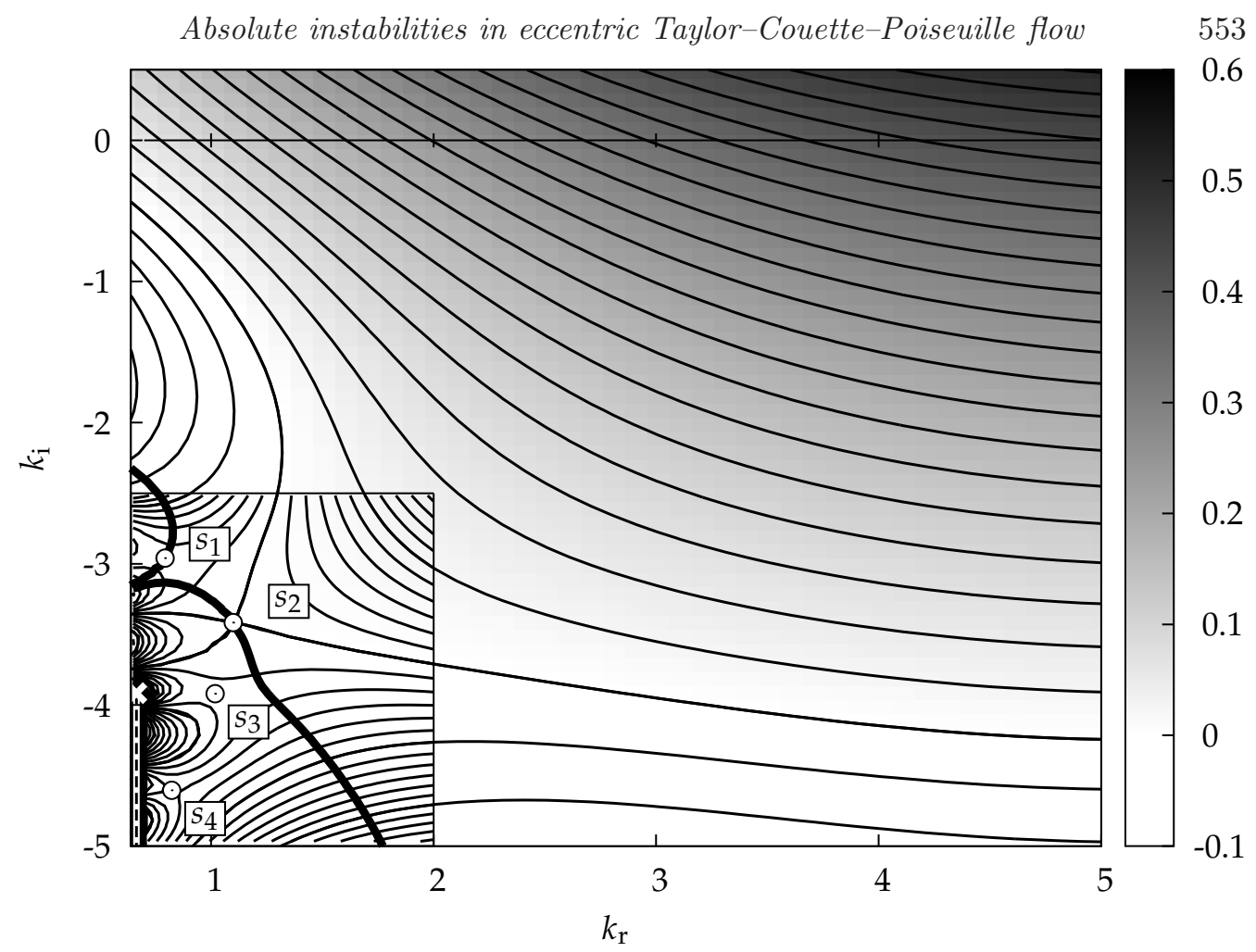

FiguRE 6. Contours of temporal growth rate at criticality for $m=0, e=0.3075$ and $R e_{z}=60$ $\left(R e_{\Omega}=472.24\right)$. Saddles are indicated by white dots. The thick line indicates the steepest descent path associated with the pinch points $s_{1}$ and $s_{2}$. The white cross indicates a branch point $\partial k / \partial \omega=0$ from which originates a branch cut (vertical dotted line). The temporal growth rate of $s_{2}$ is larger than that of $s_{1}$ : the pinch $s_{2}$ dominates the impulse response.
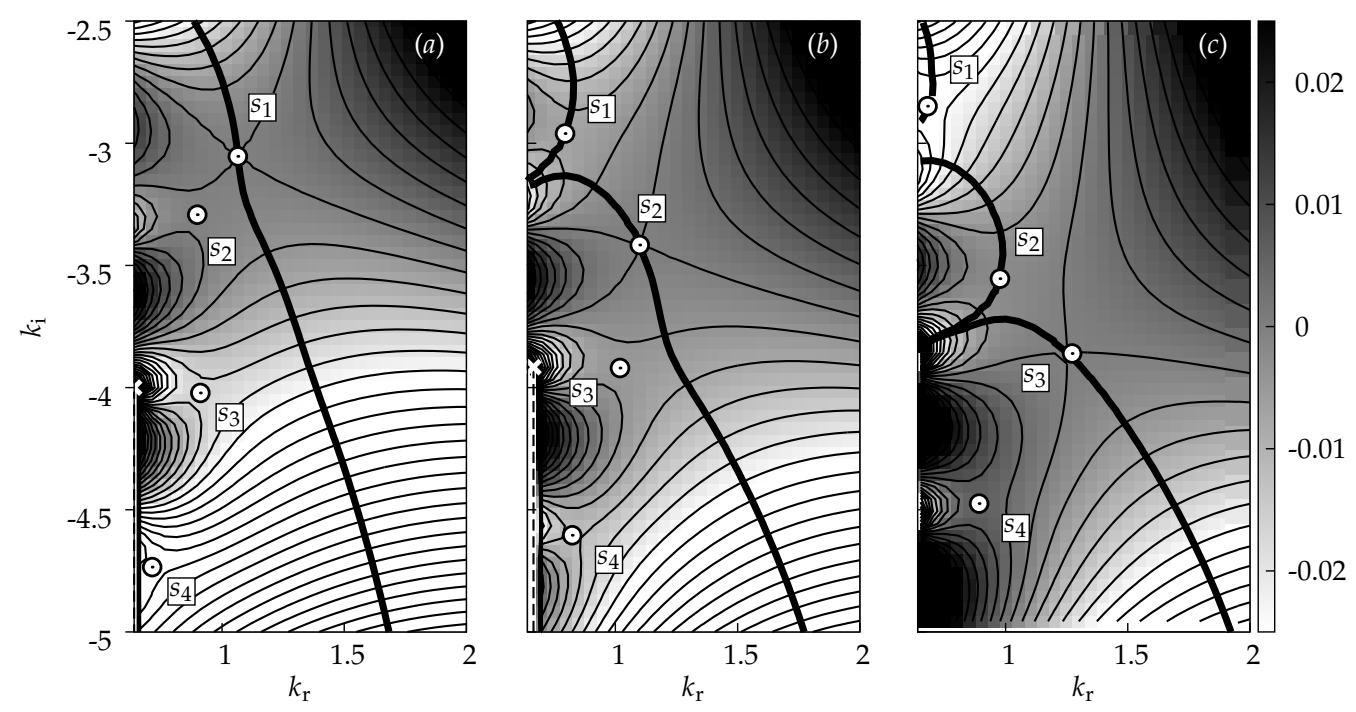

FiguRE 7 . Zoom of the bottom-left corner of figure 6 for the same value of $R e_{z}$, three eccentricities around $e \approx 0.3$ and their associated critical $\operatorname{Re}_{\Omega}$; (a) $e=0.285, \operatorname{Re}_{\Omega}=471.65, s_{1}$ is the dominating pinch; (b) zoom of previous figure, $s_{2}$ dominates; (c) $e=0.33, R_{\Omega}=468.00, s_{3}$ dominates. 


\subsection{Critical azimuthal Reynolds number}

In figure 8, the critical azimuthal Reynolds number is represented as a function of eccentricity, for $R e_{z}=0,10, \ldots, 60$. We start by describing the curves in terms of $m$. Solid lines correspond to $m \geqslant 0$, and dotted lines are for $m<0$. Changes of critical saddle point are indicated with a filled (resp. open) circle for $m \geqslant 0$ (resp. $m<0$ ). The most important result is that for any value of $e$ and $R e_{z}, m=0$ always has the lowest critical $R e_{\Omega}$, followed by $|m|=1$, and finally $|m|=2$. This observation gives credence to the assumption that modes with $|m| \geqslant 3$ can be ignored in the analysis. Curves 8(b) and 8(c) also prove the importance of considering both positive and negative $m$, as LH are not always more absolutely unstable than RH. Indeed, for low eccentricities, RH1 is slightly more unstable than LH1, but the converse is true for high eccentricities. The dynamics is even more subtle for $|m|=2$, as LH2 are generally more unstable than RH2, except for a small range of eccentricities that varies with $R e_{z}$.

The effect of axial advection is to stabilize all the modes. For high enough $R e_{z}$, the critical $R e_{\Omega}$ seems to increase almost linearly with $R e_{z}$. The rate of increase is much stronger for low eccentricities than for high eccentricities, regardless of the value of $m$, as already mentioned. Critical $R e_{\Omega}$ are typically one order of magnitude higher than $R e_{z}$, which means that the inner cylinder must be rotated much faster than the mean axial velocity to have self-sustained oscillations. Indeed, axial flow prevents AI by carrying perturbations downstream while rotation amplifies them.

The effect of eccentricity is more complex than that of $R e_{z}$. For low eccentricities, $R e_{\Omega}$ increases slowly for $m=0,1$, but decreases for all other modes. For high enough $e$, all the curves have the same shape: $R e_{\Omega}$ decreases before reaching a minimum and then increases again beyond this minimum. TV and LH1 display another similarity: they switch critical saddle point between $0.2 \leqslant e \leqslant 0.4$, for high enough $R e_{z}$. This change of saddle point coincides with the change in sign of the slope: critical $R e_{\Omega}$ increases with $e$ for the first saddle point, but decreases for the second one. As was already mentioned in paragraph $\S 3.2$, TV even changes pinch point twice for $R e_{z}=60$. On the other hand, the critical curves for RH1 are smooth, because they are obtained by continuously following a single saddle point. Finally, two saddle points define the critical curves of LH2 and RH2. For low values of $R e_{z}$ and $e$, the AI threshold is quite complex, and the curves display folds. Folds in the critical curves mean that there are finite ranges of AI in parameter space, surrounded by CI. This unusual behaviour has already been pointed out by Altmeyer et al. (2011) in the concentric case, but it seems important here to underline the fact that for high enough $R e_{\Omega}$, all the modes eventually become absolutely unstable, regardless of any 'island' of AI occurring at lower $R_{\Omega}$ (cf. figure 5).

\subsection{Critical absolute wavenumber $k_{0, \mathrm{r}}$}

Figure 9 shows the evolution of the absolute wavenumber $k_{0, \mathrm{r}}$, spatial growth rate $-k_{0, \mathrm{i}}$ and frequency $\omega_{0, \mathrm{r}}$ associated with the dominant mode $m=0$, at critical conditions. As before, curves are plotted as functions of $e$, for $R e_{z}=0,10, \ldots, 60$. The absolute wavenumber $k_{0, \mathrm{r}}$ (figure $9(a)$ ) evolves in different ways below and above $e \approx 0.3$. Below $e \approx 0.3$, critical modes have longer wavelengths as $R e_{z}$ increases, spanning up to 6 times the clearance for $R e_{z}=60$ and $e \approx 0.3$. When $e$ is high enough, the trend is the opposite, and critical modes have shorter wavelengths as $R e_{z}$ increases. Below $e \approx 0.3$, $k_{0, \mathrm{r}}$ is almost constant, or slightly decreasing with $e$, whereas above $e \approx 0.3$, it is clearly increasing with $e$. For large enough $e$, the critical wavelength seems to be controlled by the smaller clearance $d(1-e)$. Small discontinuities in $k_{0, \mathrm{r}}$ around $e \approx 0.3$ indicate a change of critical saddle point.

Comments on the curves obtained for other $m$, although not displayed here for clarity, 
Absolute instabilities in eccentric Taylor-Couette-Poiseuille flow
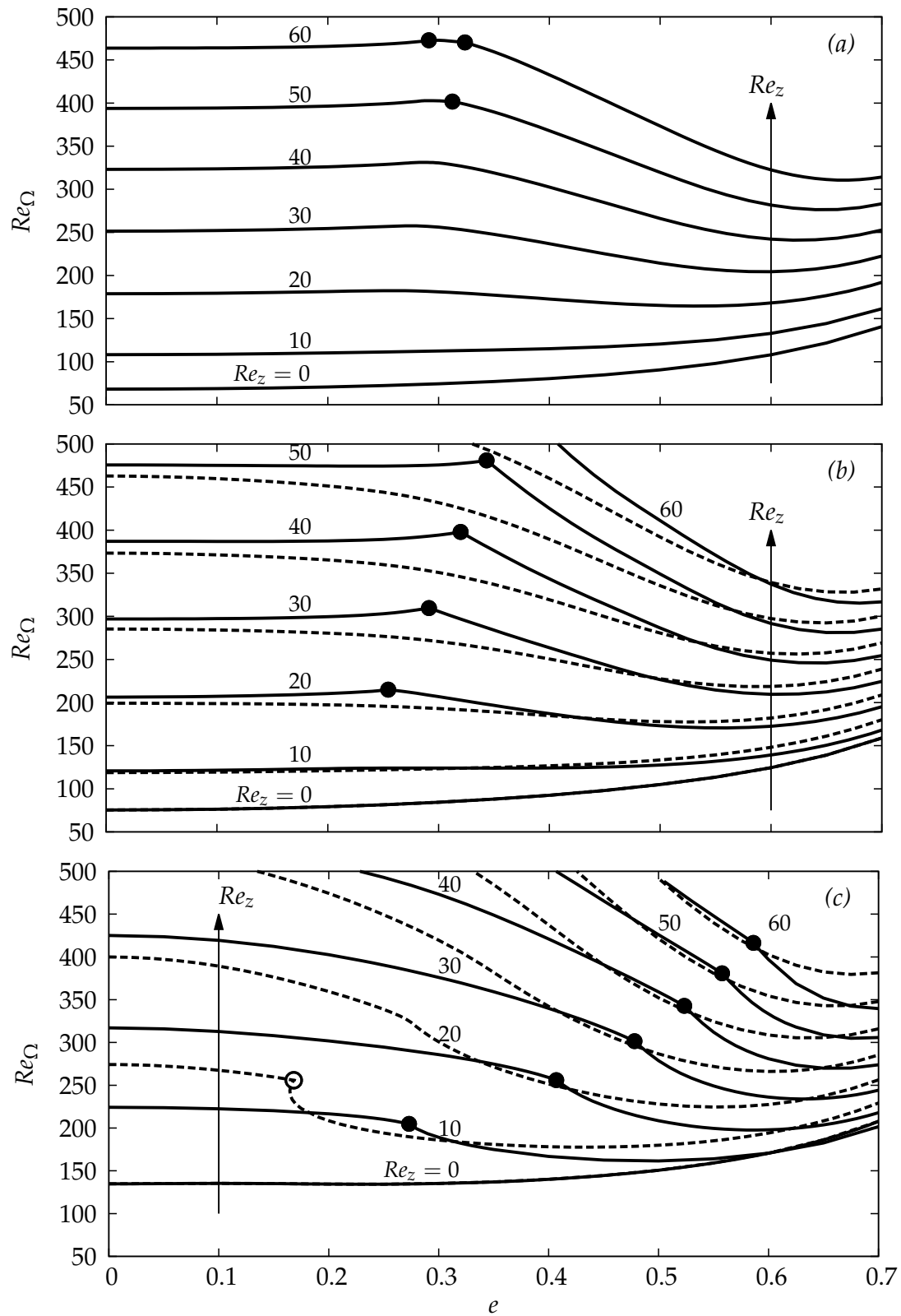

Figure 8. Critical azimuthal Reynolds number $R e_{\Omega}$ versus eccentricity $e$ for (a) $m=0$, (b) $|m|=1$ and (c) $|m|=2$. Curves are drawn for $R e_{z}=0,10, \ldots, 60$. Solid lines are for $m \geqslant 0$, and dotted lines for $m<0$. Filled/open dots indicate a change of saddle point.

can be made. First, curves of $k_{0, \mathrm{r}}$ for LH1 are very similar to those of TV, except that LH1 only changes saddle point once for $R e_{z}=60$ instead of twice for TV. Ignoring the change of saddle point, they are also qualitatively similar to those of RH1. For all $m, k_{0, \mathrm{r}}$ always increases with $e$ for high enough eccentricities, showing that all modes scale with the small gap at critical conditions. For $|m|=2$, large values of $k_{0, \mathrm{r}}$ up to 8 and more are obtained for low eccentricites as well, provided $R e_{z}$ is high enough. This behaviour is not found for other modes, where $k_{0, \mathrm{r}}$ is always between 1 and 3.5 when $e \lesssim 0.3$. For LH2 

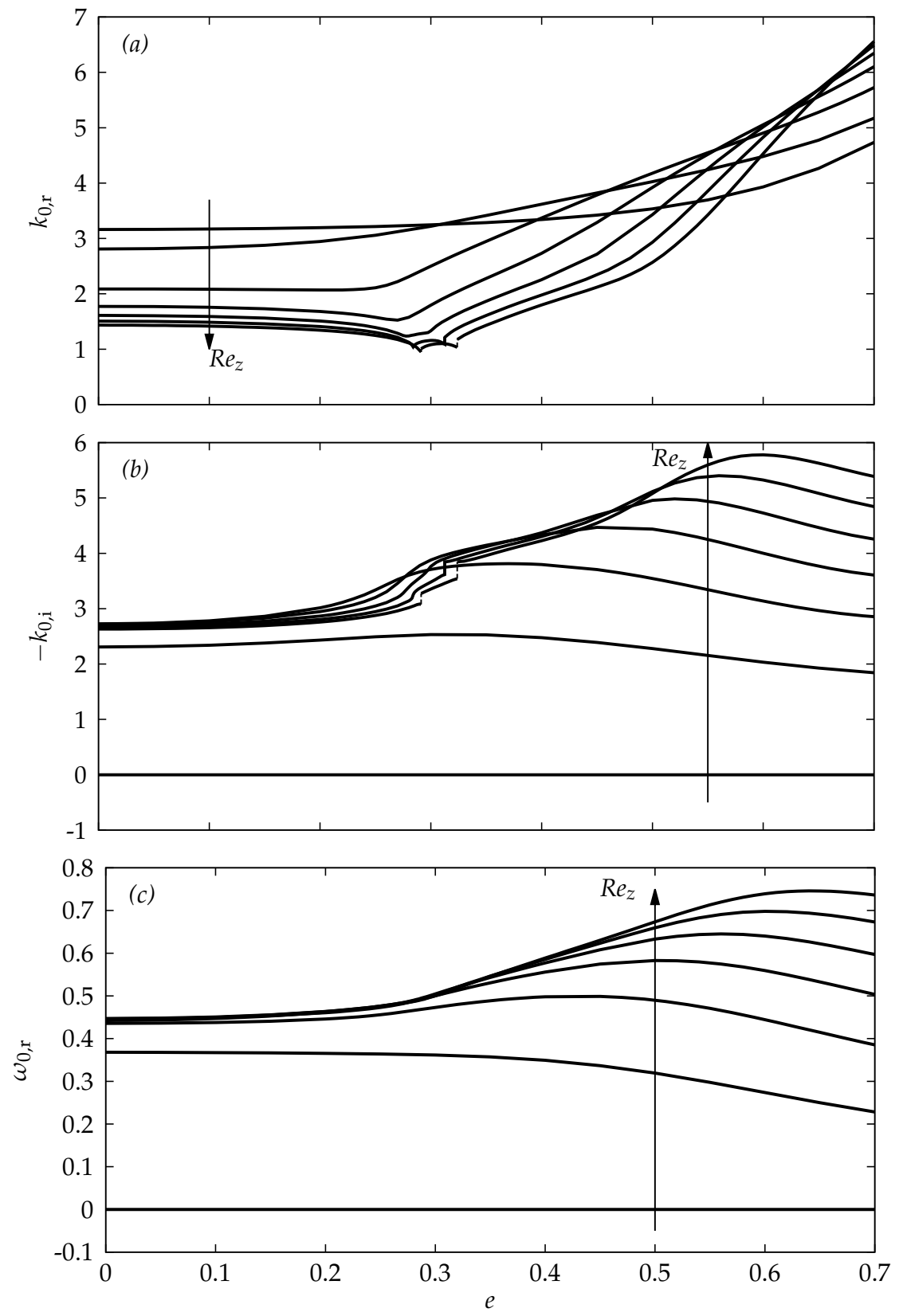

Figure 9. (a) Absolute wavenumber $k_{0, \mathrm{r}}$, (b) spatial growth rate $-k_{0, \mathrm{i}}$ and (c) frequency $\omega_{0, \mathrm{r}}$ for $m=0$ at critical conditions, versus eccentricity $e$. Curves are given for $R e_{z}=0,10, \ldots, 60$.

and RH2, a large discontinuity of axial wavenumber is observed upon switching saddle point. For example, $k_{0, \mathrm{r}}$ of LH2 varies from about 7.5 to about 3.5 for $R e_{z}=60$ and $e \approx 0.6$.

\subsection{Critical absolute spatial growth rate $-k_{0, \mathrm{i}}$}

The absolute spatial growth rate $-k_{0, \mathrm{i}}$ (figure $\left.9(b)\right)$ measures the 'steepness' of the stationary front of the impulse response wavepacket. For $e \lesssim 0.3$, it is slightly increasing 
with $e$. It varies quickly for $R e_{z} \lesssim 20$, and then slowly varies in the range $2.5 \lesssim-k_{0, \mathrm{i}} \lesssim 4$ for higher $R e_{z}$. For $e \gtrsim 0.3,-k_{0, \mathrm{i}}$ increases with $e$, reaches a maximum value, and then decreases again. For high enough $e,-k_{0, \mathrm{i}}$ increases steadily with $R e_{z}$.

Similar trends are noticed for LH1 and RH1, with comparable ranges of values. For LH2 and RH2 however, the curves are quite different. For low eccentricities, $-k_{0, \mathrm{i}}$ increases significantly with $R e_{z}$, whereas it is almost constant for other $m$. Therefore, extreme front steepness occurs for LH2 at low $e$, with $-k_{0, \mathrm{i}}>12$ for $R e_{z}=60$.

For RH1 and RH2 at low $R e_{z},-k_{0, i}$ can be negative over the whole range of eccentricities. Physically, this means that the stationary front is the leading one in this case $V_{+}=0$ (cf. section $\S 2.4$ ), and that the most temporally unstable RH wave has a negative group velocity.

Finally, a common feature of all $m$ is that $-k_{0, \mathrm{i}}$ has a maximum as a function of $e$.

\subsection{Critical absolute frequency $\omega_{0, \mathrm{r}}$}

The absolute frequency $\omega_{0, r}$ (figure $9(c)$ ) is given here for reference. After nonlinear saturation of the instability, self-sustained oscillations with frequency close to $\omega_{0, r}$ are expected for a supercritical transition. Therefore, the values of $\omega_{0, r}$ can be used as a good estimate of the hydrodynamic resonance frequency of the flow, and may be of interest for engineering applications. The trends of the curves are very similar to those obtained for the spatial growth rate. The frequency range is shifted towards higher values as $m$ increases. For RH1 and RH2, $\omega_{0, r}$ can be negative (always the case for RH2), indicating that the absolute phase speed $c_{0} \equiv \omega_{0, \mathrm{r}} / k_{0, \mathrm{r}}$ of the mode is negative in this case. Finally, discontinuities in the absolute frequency occur for all modes but RH1, because of changes in critical saddle point. Discontinuities are not clearly visible for $m=0$ and the change of saddle point is 'smooth', as will be discussed later in $\S 4.3$.

\subsection{Absolute temporal growth rate $\omega_{0, \mathrm{i}}$ maps}

Critical curves in $\S 3.3$ indicate the AI domain for each $m$. However, they do not indicate which mode will be the most absolutely unstable if $R e_{\Omega}$ is above two or more thresholds. Indeed, the mode which bifurcates first as $R e_{\Omega}$ is increased does not necessarily have the highest absolute growth rate $\omega_{0, \mathrm{i}}$ for larger driving. Figure 10 represents isocontours of $\omega_{0, \mathrm{i}}$ in $R e_{\Omega^{-}} R e_{z}$ plane for $m=0,1,2$, and $e=0,0.2,0.7$. Curves for $m=-1,-2$ can be recovered upon applying the $\Pi_{3}$-symmetry $(2.5)$.

The first conclusion is that $m=0$ remains the most unstable mode over the whole range of parameters. However, for $e=0.7$, isocontours of $m=1$ are very close to those for $m=0$, and one may expect LH1 to become more absolutely unstable than TV for larger eccentricities and large $R e_{z}$.

Isocontours of $\omega_{0, \mathrm{i}}$ for $m=1$ and $m=-1$ (using $\Pi_{3}$-symmetry) are generally close. For low eccentricity, RH1 is always more unstable than LH1, but for high eccentricity, the converse is true. For a moderate eccentricity of $e \approx 0.5$ (not shown here), the dominant mode depends on the specific values of $R e_{\Omega}$ and $R e_{z}$.

For $m=1,2$, some isocontours have discontinuous slopes, as a consequence of a change of dominant saddle point. In figure 10 (3a), the 'island' of instability previously presented in figure 5 is shown to be connected to a 'continent', for higher values of $R e_{\Omega}$, via a change of critical saddle point. Indeed, in their analysis of $m=2$ in the axisymmetric case, Altmeyer et al. (2011) restricted their analysis to the reference saddle point defined in $\S 3.1$, even though the authors mentioned the existence of other saddle points. Figure 10(3a) gives the complete AI boundary for this case. For higher eccentricities, the 'continent' of instability absorbs the 'island', and for $e=0.7$, the saddle point associated to the 'island' is always sub-dominant. When considering the saddle point associated with the 
'continent', LH2 is generally more unstable than RH2. On the other hand, for low $e$ and very low $R e_{z}, \mathrm{RH} 2$ can be more unstable than LH2 because of the 'island' of instability. For moderate eccentricities, the ordering depends on the specific values of $R e_{\Omega}$ and $R e_{z}$.

\section{Discussion}

In this section, we study the critical modes and the production of perturbation kinetic energy. We discuss the results and the instability mechanism in the light of these elements.

\subsection{Critical modes}

In figure 11(1,2), we examine the spatial distribution of the mode $m=0$ at critical conditions for $R e_{z}=60$ and three representative eccentricities: $e=0.2,0.4,0.7$. The three-dimensional distribution of axial velocity is shown, together with the corresponding time-averaged distribution of perturbation kinetic energy $\mathcal{E}=\frac{1}{2}\left(\left\|\tilde{\mathbf{u}}_{\perp}\right\|^{2}+|\tilde{w}|^{2}\right)$ (the $\exp \left[-k_{\mathrm{i}} z\right]$ dependence of the mode amplitude is omitted).

Consider the polar angle $\theta$ of the maximum of energy, with respect to the inner cylinder center, indicated in figure 2. For low eccentricities or low $R e_{z}$, the mode is localised in the wide gap, at positive $\theta$. However, for larger $e$ or $R e_{z}$, this maximum is shifted upstream to the region $-90^{\circ} \leqslant \theta \leqslant 0^{\circ}$. For $e=0.7$, the mode concentrates in the vicinity of the inner cylinder, on the wide gap side, but has a radial extent scaling with the small gap. When $e$ increases for $R e_{z}=60$, the ratio between the maximum, and the average value increases from less than 2 for $e=0$ to almost 14 for $e=0.7$, indicating confinement of the mode into a smaller region of the annulus. Finally, the only contribution of $\tilde{\mathbf{u}}_{\perp}$ to $\mathcal{E}$ accounts for $65 \%$ to $85 \%$ of the total perturbation energy when $e$ increases from 0 to 0.7 .

Similar behaviour is found for LH1 at $R e_{z}=60$. For RH1 however, the energy is less tightly concentrated. The maximum of $\mathcal{E}$ still occurs at negative angles, but does not go beyond $-45^{\circ}$ in this case. It is concluded that the appareance of the peak of perturbation energy at large negative angles is associated with the change of saddle point occurring for both TV and LH1.

Surprisingly, the critical modes of AI and CI peak at completely different regions of the annulus for moderate eccentricities and high $R e_{z}$. In comparison (cf. Leclercq et al. (2013)), the maximum energy of the critical mode of CI for $e=0.5$ is always localised at large positive angles. No direct comparison should be made with AI because critical modes of CI are obtained for different threshold values of $R e_{\Omega}$. It is nonetheless interesting to observe that modes can be localised at either positive or negative polar angles depending on the situation.

Finally, we enumerate characteristics common to all $m$. First, in-plane motion accounts for the larger contribution to the total perturbation kinetic energy of all $m$ for $R e_{z}=60$. Also, as $e$ approaches 0.7 for $R e_{z}=60$, all $m$ tend to have similar distributions of energy, with strong localisation close to the inner cylinder, over a radial extent scaling with the small gap. In addition, we recall that the critical wavenumber of all modes takes on large values $k_{0, \mathrm{r}} \sim 6.5-8$ when $e=0.7$ and $R e_{z}=60$. These observations indicate that for high eccentricities, the critical modes for all $m$ scale with the small gap.

\subsection{Production of perturbation kinetic energy}

To further investigate the instability mechanism, the production of perturbation kinetic energy is calculated. In the well-known Reynolds-Orr equation, the local rate of production of $\mathcal{E}$ is given by $-\mathbf{u}^{\prime} \cdot\left(\mathbf{u}^{\prime} \cdot \boldsymbol{\nabla} \mathbf{U}\right)$. Averaging in time and separating velocities into in-plane and axial components, one can define two contributions (the spatial growth of 


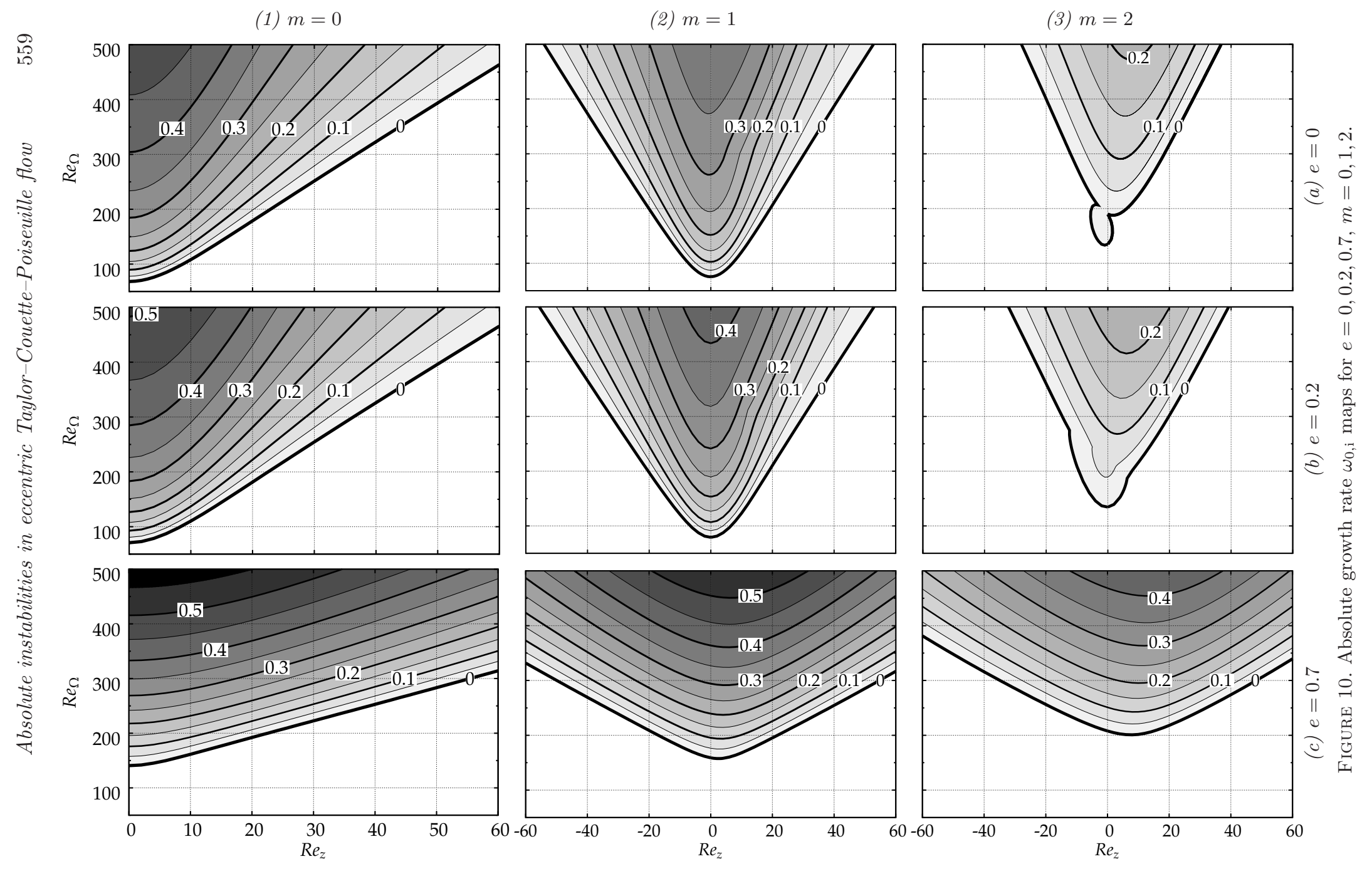




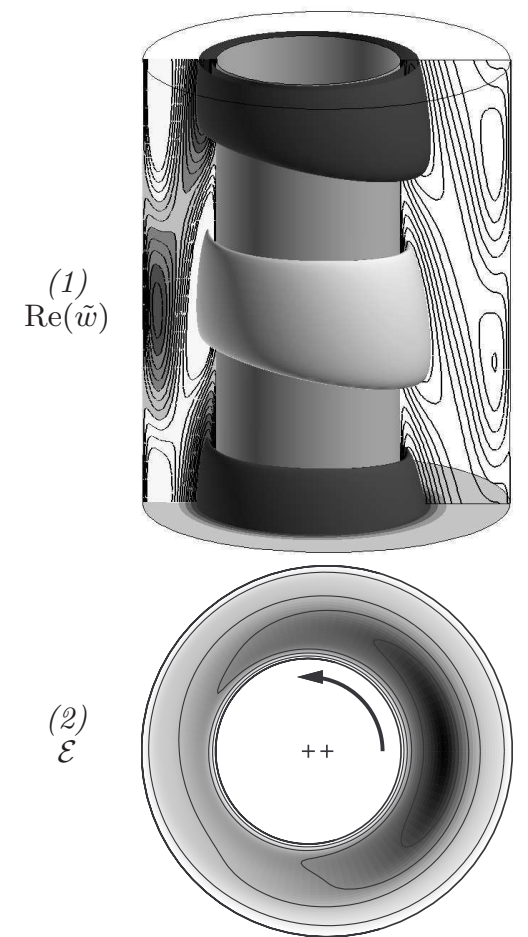

(a) $e=0.2$
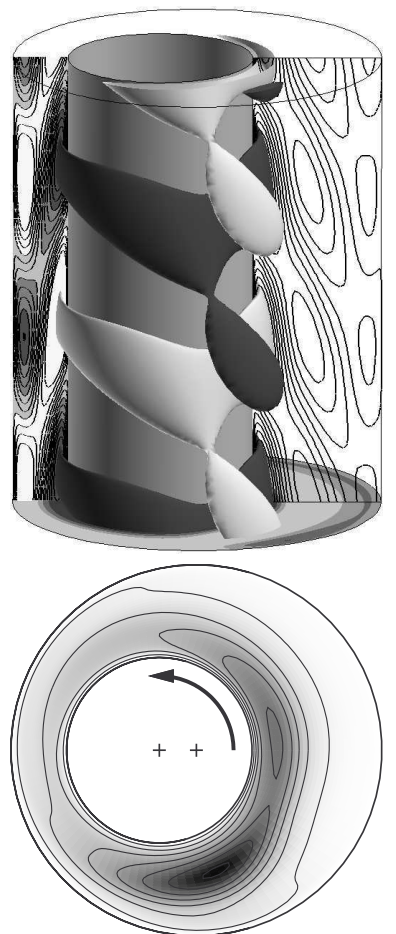

(b) $e=0.4$
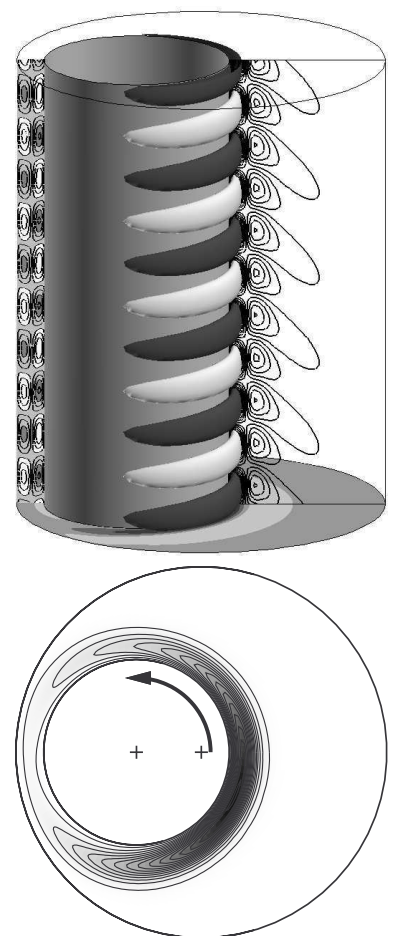

(c) $e=0.7$

Figure 11. Critical mode $m=0$ for $R e_{z}=60$, (a) $e=0.2, R e_{\Omega}=465.82$, (b) $e=0.4$, $R e_{\Omega}=432.74$, (c) $e=0.7, R e_{\Omega}=314.16$. (1) Distribution of axial perturbation velocity $\operatorname{Re}(\tilde{w})$. Dark (resp. light) grey is for positive (resp. negative) values. (2) Distribution of perturbation kinetic energy $\mathcal{E}=\frac{1}{2}\left(\left\|\tilde{\mathbf{u}}_{\perp}\right\|^{2}+|\tilde{w}|^{2}\right)$. Dark grey indicates high values, and isocontours are equispaced.

the mode amplitude along $z$ is ignored again)

$$
\mathcal{P}_{\perp}=-\frac{1}{2} \operatorname{Re}\left\{\tilde{\mathbf{u}}_{\perp}^{\star} \cdot\left(\tilde{\mathbf{u}}_{\perp} \cdot \nabla_{\perp} \mathbf{U}_{\perp}\right)\right\} \text { and } \mathcal{P}_{z}=-\frac{1}{2} \operatorname{Re}\left\{\tilde{w}^{\star}\left(\tilde{\mathbf{u}}_{\perp} \cdot \nabla_{\perp} W\right)\right\}
$$

corresponding to the work of the Reynolds stresses against the in-plane and axial shear respectively. Expressions for the nonlinear terms in the modified bipolar coordinate system are given in Leclercq et al. (2013). Because the basic flow is axially invariant, these two terms are the only contributions to the production of $\mathcal{E}$.

Figure 12 represents the distribution of $\mathcal{P}_{\perp}$ and $\mathcal{P}_{z}$ for $m=0$ and $R e_{z}=60$, at $e=0.2,0.4,0.7$, as in figure 11 . Only positive contributions are shown in grey shades, as negative contributions inhibit temporal growth. Distributions of $\mathcal{P}_{\perp}$ and $\mathcal{E}$ look very similar: $\mathcal{P}_{\perp}$ is maximum in the wide gap for low $e$, then at negative polar angles for moderate $e$, then close to the inner cylinder on the wide gap side for $e$ close to 1 . The dominant contribution to $\mathcal{P}_{\perp}$ comes from the Reynolds stress term involving the pseudoradial derivative of the azimuthal velocity $V$. The same calculation of $\mathcal{P}_{\perp}$ and $\mathcal{P}_{z}$ has been performed for the critical mode of the CI at $R e_{z}=60$ and $e=0.5(m=2)$. $\mathcal{P}_{\perp}$ was also found to account for most of the kinetic energy production (81\%), but the peak was located at a positive polar angle of $92^{\circ}$.

Distributions of $\mathcal{P}_{z}$ show larger contributions near the walls, where $\left\|\nabla_{\perp} W\right\|$ is larger. As eccentricity increases, production of kinetic energy close to the outer cylinder decreases, as the mode concentrates in the vicinity of the inner cylinder. For $e=0.7, \mathcal{P}_{z}$ 


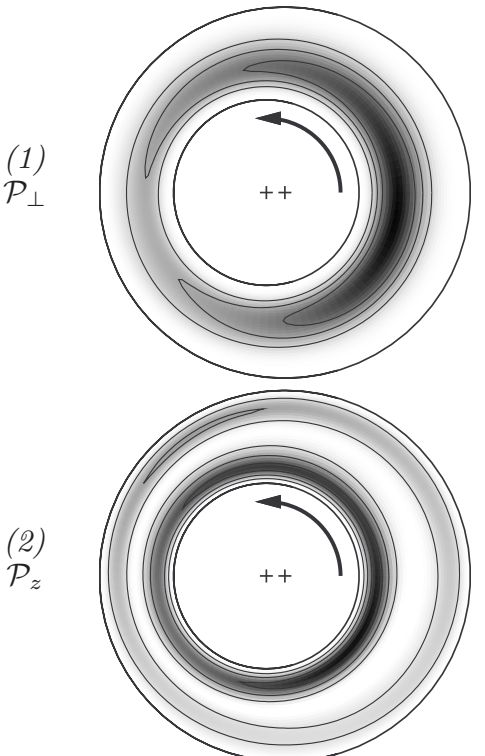

(a) $e=0.2$

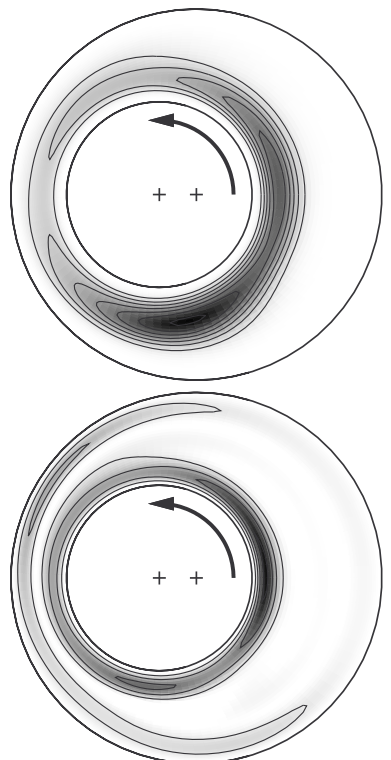

(b) $e=0.4$

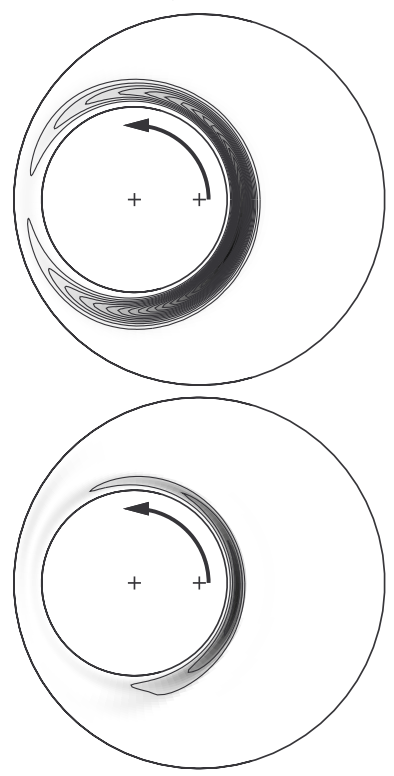

(c) $e=0.7$

Figure 12. Distribution of kinetic energy production for $m=0$ and $R e_{z}=60$, at critical conditions. (1) $\mathcal{P}_{\perp}$ and (2) $\mathcal{P}_{z}$. (a) $e=0.2, R e_{\Omega}=465.82$, (b) $e=0.4, R e_{\Omega}=432.74$, (c) $e=0.7, \operatorname{Re}_{\Omega}=314.16$. Dark grey indicates large contributions and white corresponds to zero or negative contribution. Isocontours are equispaced.

peaks almost in the same region as $\mathcal{P}_{\perp}$ and $\mathcal{E}$, namely close to the inner cylinder in the wide gap.

Integration of $\mathcal{P}_{\perp}$ and $\mathcal{P}_{z}$ over the annular domain for $R e_{z}=60$ indicates that inplane shear dominates the production of kinetic energy, $\mathcal{P}_{\perp}$ always accounting for more than $85 \%$ of the total amount, and even more than $97 \%$ for $e=0.7$. The dominant contribution comes from the term involving pseudo-radial variations of pseudo-azimuthal velocity, characteristic of a centrifugal instability.

\subsection{Instability mechanism}

The striking destabilisation occuring at moderate eccentricities remains difficult to explain even after examining the basic flow, the critical mode and the production of kinetic energy. The transition from a stabilising to a destabilising effect of eccentricity occurs around $0.2 \leqslant e \leqslant 0.4$ for both TV and LH1, through a change of critical saddle point when $R e_{z}$ is large enough. The recirculation region appears for $e \approx 0.2$ for $R e_{\Omega} \approx 500$ so it seems that the change of behaviour occurs after the recirculation zone has reached a critical size. The basic flow is then significantly different from a classical Couette flow. Indeed, while energy of the mode mostly localises in the vicinity of the inner cylinder, it also partially spans over the recirculation region for moderate eccentricities (cf. figure 11(b)). Surprisingly, a region of the flow located at negative polar angles seems to drive AI for moderate eccentricities, whereas kinetic energy production always peaks at positive angles for CI at criticality. Arguments based on local stability of the flow, however tempting in a quest of explanation, should be avoided here because of strong non-parallelism of the basic flow in the pseudo-azimuthal direction. Indeed, assuming the flow locally parallel in $\phi$ leads to completely wrong predictions of instability thresholds of eccentric Taylor-Couette flow (DiPrima 1963; Ritchie 1968). The most temporally unstable velocity profile, theoretically located at $\phi=\theta=0^{\circ}$, does not coincide with the 
location of maximum vortex activity found in the experiments (Vohr 1968). On the other hand, global analyses yield good results in this geometry (DiPrima \& Stuart 1972, 1975; Eagles et al. 1978), showing the limits of the local approach. Therefore, localisation of the modes and kinetic energy production at moderate eccentricities should be regarded as a global property of the entire flow field.

The consecutive switchovers of dominant saddle point for $e \approx 0.3$ and $R e_{z}=60$ occur very near collisions of the distinct pinches into third-order saddle points (Davies 1989) or 'super branch points' (Healey 2004), satisfying simultaneously $\partial \omega / \partial k=0$, $\partial^{2} \omega / \partial k^{2}=0$ and $\omega_{\mathrm{i}}=0$. Indeed, $s_{1}$ and $s_{2}$ collide while being neutral for $e=0.2839$, $R e_{z}=50.115, R e_{\Omega}=403.21$ while $s_{2}$ and $s_{3}$ coalesce with $\omega_{\mathrm{i}}=0$ for $e=0.3032$ and $R e_{z}=43.188, R e_{\Omega}=353.60$. As a result, the saddle points swap dominance but the absolute wavenumber, frequency, and spatial growth rate vary almost continuously through the exchange (see figures 9a,b,c). Consequently, the spatial distribution of the critical mode is little changed and the physical reason for the switchover remains unclear.

As $e$ approaches 1, all $m$ tend to behave in a similar way. After reaching a minimum value, the critical $R e_{\Omega}$ increases again as $e$ becomes larger. Instability thresholds of all $m \geqslant 0$, and even their respective absolute growth rate for any $R e_{z}-R e_{\Omega}$ combination, become close at high $e$ and less sensistive to variations of $R e_{z}$. More similarities are found by inspecting the critical modes. For $R e_{z}=60$, all $m$ have absolute wavenumbers $k_{0, \mathrm{r}}$ in the range $6.5-8$, indicating a similar length scale. Indeed, the kinetic energy of all these modes is localised around the inner cylinder, on a radial extent of the order of the smallest gap $d(1-e)$, consistent with a small wavelength. The localisation is so strong that the difference between toroidal or helical structure of the modes is partially 'blurred'. These similarities between $m$ at high $e$ is reminiscent of small gap Taylor-CouettePoiseuille flow, where critical thresholds associated with different $m$ are very close (Ng \& Turner 1982; Leclercq et al. 2013). Indeed, as eccentricity increases, the Couette-like flow associated with rotation of the inner cylinder scales as $d(1-e)$, curvature effects become less important, and $m$ behaves more and more like a continuous real wavenumber, as when $\eta \rightarrow 1$. Matching between localisation of the modes and the Couette-like part of the basic flow may also explain the weaker effect of axial advection on the instability thresholds. Indeed, for large eccentricities, most of the axial volume flux passes through the wide gap, and the maximum value of $W$ is located in the recirculation region, far from the inner cylinder. Hence, the region where perturbations are most amplified is spatially separated from the region where they are most rapidly 'blown away'. This observation could explain why critical $R e_{\Omega}$ are less sensitive to $R e_{z}$ for $e$ close to one.

\subsection{Convective versus absolute instability}

We conclude this section by comparing the thresholds of CI (Leclercq et al. 2013) and $\mathrm{AI}$ in the eccentric Taylor-Couette-Poiseuille flow. Figure 13 represents the critical $R e_{\Omega}$ for CI (dotted lines), and for AI (solid lines), as a function of $R e_{z}$ for $e=0,0.1, \ldots, 0.7$. For $R e_{z}=0$, the two thresholds for $m=0$ coincide, as expected for a steady bifurcation. The critical $R e_{\Omega}$ increases with $R e_{z}$ for both CI and AI, but with a much larger rate for AI. This was expected as axial advection tends to carry the perturbations away from the source, so a larger driving is required to reach AI. The critical mode is always $m=0$ for AI, corresponding to closed pseudo-toroidal Taylor vortices, propagating when $R e_{z} \neq 0$. For CI, LH modes of increasing $m$ become critically unstable as $R e_{z}$ increases. Open circles indicate a change of critical $m$ on the CI thresholds. The effect of eccentricity is clearly stabilising for $\mathrm{CI}$, but the effect on $\mathrm{AI}$ is more complex. For high enough $R e_{z}$, as eccentricity increases, the critical $R e_{\Omega}$ of AI slightly increases for low $e$, but then decreases before reaching a minimum and increases again for larger values of $e$. 


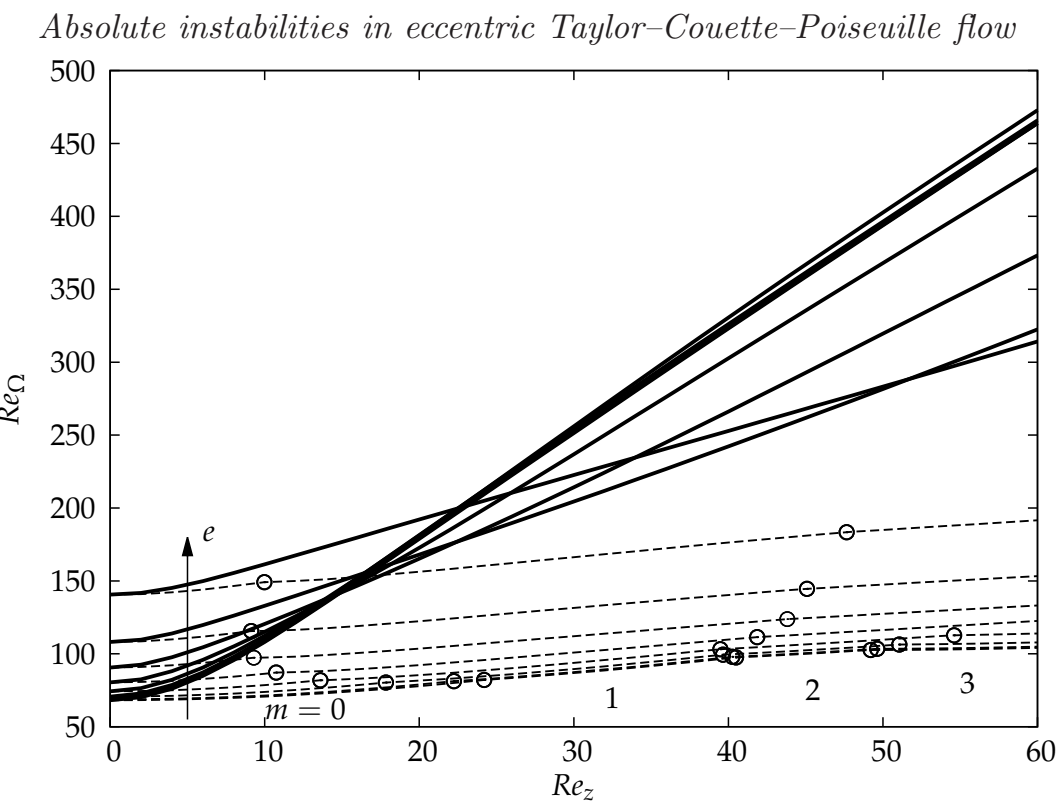

Figure 13. Absolute (solid lines) and convective (dotted lines) instability thresholds: critical $R e_{\Omega}$ versus $R e_{z}$, for $e=0,0.1, \ldots, 0.7$. Open circles indicate a change of critical $m$ for CI. For $\mathrm{AI}$, the critical mode is always $m=0$.

\section{Conclusions}

In this paper, an absolute instability analysis has been performed for the flow between eccentric cylinders, with rotation of the inner one and a superimposed pressure-driven axial flow. The ratio between cylinder radii was fixed at $\eta=0.5$, a value representative of an oil-well-drilling configuration. Five modes of instability have been considered, $m=$ $-2,-1,0,1,2$, thus including the most temporally unstable modes up to $R e_{z}=50$ found in Leclercq et al. (2013). Throughout the range of $R e_{z}$ and $e$ considered in the present study, the mode with the largest absolute growth rate is always the pseudo-toroidal vortex flow corresponding to $m=0$. Unlike the temporal growth rate, the absolute growth rate of left-handed pseudo-helical modes $(m>0)$ is not always larger than that of right-handed ones $(m<0)$.

Increasing $R e_{z}$ tends to hinder absolute instability because axial flow sweeps perturbations downstream. As a rule of thumb, the rotational velocity of the inner cylinder needs to be approximately one order of magnitude larger than the mean axial velocity to trigger absolute instability.

The effect of eccentricity is more complex and increasing $e$ can result in destabilisation for large enough $R e_{z}$ and moderate eccentricities $0.3 \lesssim e \lesssim 0.6$. In this case, the critical mode has a complex structure, and the production of kinetic energy peaks at a welldefined region of the annulus, located in the diverging gap region. Outside this range of eccentricities, increasing $e$ has a stabilising effect, increasingly so as the limit of touching cylinders is approached.

The instability mechanism is purely centrifugal in nature and the critical-mode axial wavelength and radial extent scale as the smallest gap $d(1-e)$. For large eccentricities, all the modes localise in the vicinity of the inner cylinder, and their spatial distributions become more and more similar. The effect of $R e_{z}$ on absolute instability thresholds becomes weaker, and the distance between thresholds associated to different $m$ diminishes.

Overall, many valid saddle points were found for the different instability modes, and 
the critical pinch point switches upon varying the flow parameters. The physical interpretation of the switchovers of saddle point occuring near $e \approx 0.3$ for $m=0$ and $R e_{z}=60$ is unclear, because the saddle points are associated with modes having almost the same spatial distribution and spatio-temporal properties. Indeed, when they swap, the saddle points almost collide into third-order saddle points, where they would be impossible to distinguish.

We believe that the most crucial outlook of this work is additional experiments to confirm our findings on convective and absolute instabilities, since the last measurements made on such a configuration are older than the introduction of absolute instability theory to fluid mechanics (Huerre \& Monkewitz 1985)! Even in the convectively unstable régime, the literature is very lean, and only one apparatus seems to have ever existed, whereas the domain of application is vast. On a theoretical viewpoint, it would be particularly interesting to investigate the properties of nonlinear global modes, partly based on local absolute instability properties (Pier et al. 2001), when eccentricity varies slowly along the axis. Indeed, bending of the long drillstring results in axially varying eccentricity. Moreover, such weakly non-parallel open flows are believed to be good candidates to confirm the potential existence of hat modes (Pier \& Huerre 1996, 2001), theoretically predicted for model equations, but yet to be identified in a real configuration.

\section{REFERENCES}

Altmeyer, S., Hoffmann, C. \& LüCKe, M. 2011 Islands of instability for growth of spiral vortices in the Taylor-Couette system with and without axial through flow. Phys. Rev. E 84, 046308.

Babcock, K. L., Ahlers, G. \& Cannell, D. S. 1991 Noise-sustained structure in TaylorCouette flow with through flow. Phys. Rev. Lett. 67, 3388-3391.

Babcock, K. L., Ahlers, G. \& Cannell, D. S. 1994 Noise amplification in open TaylorCouette flow. Phys. Rev. E 50, 3670-3692.

Babcock, K. L., Cannell, D. S. \& Ahlers, G. 1992 Stability and noise in Taylor-Couette flow with through-flow. Physica D 61, 40-46.

Bers, A. 1983 Space-time evolution of plasma instabilities-absolute and convective. In Handbook of Plasma Physics (ed. M. Rosenbluth \& R. Sagdeev), pp. 451-517. North-Holland.

BRIGGs, R. J. 1964 Electron-stream Interactions with Plasmas, pp. 1-46. MIT Press.

Chomaz, J.-M. 2005 Global instabilities in spatially developing flows: non-normality and nonlinearity. Annu. Rev. Fluid Mech. 37, 357-392.

Coney, J. E. R. 1971 Taylor vortex flow with special reference to rotary heat exchangers. PhD thesis, Leeds University.

Coney, J. E. R. \& Atkinson, J. 1978 The effect of Taylor vortex flow on the radial forces in an annulus having variable eccentricity and axial flow. Trans. ASME: J. Fluids Engng 100, $210-214$.

Coney, J. E. R. \& MobBs, F. R. 1969-70 Hydrodynamic stability of the flow between eccentric rotating cylinders with axial flow: visual observations (paper 2). Proc. Inst. Mech. Engrs $184 \mathrm{Pt}$ 3L, 10-17.

Couette, M. 1888a La viscosité des liquides. Bull. Sci. Phys. 4, 40-62,123-133,262-278.

Couette, M. $1888 b$ Sur un nouvel appareil pour l'étude du frottement des fluides. C. R. Acad. Sci. (Paris) 107, 388-390.

DAviEs, J. A. 1989 Conditions for absolute instability in the cyclotron resonance maser. Phys. Fluids B 1, 663-669.

DiPrima, R. C. 1960 The stability of a viscous fluid between rotating cylinders with an axial flow. J. Fluid Mech. 9, 621-631.

DiPrima, R. C. 1963 A note on the stability of flow in loaded journal bearings. Trans. Am. Soc. Lubric. Engrs 6, 249-253.

DiPrima, R. C. \& Stuart, J. T. 1972 Non-local effects in the stability of flow between eccentric rotating cylinders. J. Fluid Mech. 54, 393-415. 
DiPrima, R. C. \& Stuart, J. T. 1975 The nonlinear calculation of Taylor vortex flow between eccentric rotating cylinders. J. Fluid Mech. 67, 85-111.

DiPrima, R. C. \& Swinney, H. L. 1985 Instabilities and transition in flow between concentric rotating cylinders. In Hydrodynamic instabilities and the transition to turbulence (eds. H. L. Swinney and J. P. Gollub), pp. 139-180. Springer.

Eagles, P. M., Stuart, J. T. \& DiPrima, R. C. 1978 The effects of eccentricity on torque and load in Taylor-vortex flow. J. Fluid Mech. 87, 209-231.

Escudier, M. P., Oliveira, P. J. \& Pinho, F. T. 2002 Fully developed laminar flow of purely viscous non-newtonian liquids through annuli, including the effects of eccentricity and inner-cylinder rotation. Intl J. Heat Fluid Flow 23, 52-73.

Guo, B. \& Liu, G. 2011 Applied Drilling Circulation Systems - Hydraulics, calculations, models. Elsevier.

HeAley, J. J. 2004 On the relation between the viscous and inviscid absolute instabilities of the rotating-disk boundary layer. J. Fluid Mech. 511, 179-199.

Huerre, P. 2000 Open shear flow instabilities. In Perspectives in Fluid Dynamics (eds. G. K. Batchelor, H. K. Moffat \& M. G. Worster), pp. 159-229. Cambridge University Press.

Huerre, P. \& Monkewitz, P. A. 1985 Absolute and convective instabilities in free shear layers. J. Fluid Mech. 159, 151-68.

Huerre, P. \& Monkewitz, P. A. 1990 Local and global instabilities in spatially developing flows. Annu. Rev. Fluid. Mech. 22, 473-537.

Juniper, M. P. 2006 The effect of confinement on the stability of two-dimensional shear flows. J. Fluid Mech. 565, 171-195.

Keller, H. B. 1977 Numerical solution of bifurcation and nonlinear eigenvalue problems. In Applications of Bifurcation Theory (ed. P. Rabinowitz), pp. 359-384. Academic.

Leclercq, C., Pier, B. \& Scott, J. F. 2013 Temporal stability of eccentric Taylor-CouettePoiseuille flow. J. Fluid Mech. 733, 68-99.

Lehoucq, R. B., Sorensen, D. C. \& YAng, C. 1997 ARPACK users' guide: Solution of large-scale eigenvalue problems with implicitly restarted Arnoldi methods.

Lücke, M. \& ReckTenwald, A. 1993 Amplification of molecular fluctuations into macroscopic vortices by convective instabilities. Europhys. Lett. 22, 559 .

Mallock, A. 1888 Determination of the viscosity of water. Proc. R. Soc. Lond. 45, 126-132.

Martinand, D., Serre, E. \& Lueptow, R. M. 2009 Absolute and convective instability of cylindrical Couette flow with axial and radial flows. Phys. Fluids 21, 104102.

Mobbs, F. R. \& Younes, M. A. M. A. 1974 The Taylor vortex regime in the flow between eccentric rotating cylinders. Trans. ASME: J. Lubric. Tech. pp. 127-134.

NG, B. S. \& TuRner, E. R. 1982 On the linear stability of spiral flow between rotating cylinders. Proc. R. Soc. Lond. A Mat. 382, 83-102.

Pier, B. \& Huerre, P. 1996 Fully nonlinear global modes in spatially developing media. Physica D 97, 206-222.

Pier, B. \& Huerre, P. 2001 Nonlinear synchronization in open flows. J. Fluids Struct. 15, $471-480$.

Pier, B., Huerre, P. \& Chomaz, J.-M. 2001 Bifurcation to fully nonlinear synchronized structures in slowly varying media. Physica $D$ 148, 49-96.

Pinter, A., Lücke, M. \& Hoffmann, C. 2003 Spiral and Taylor vortex fronts and pulses in axial through flow. Phys. Rev. E 67, 026318.

Ritchie, G. S. 1968 On the stability of viscous flow between eccentric rotating cylinders. J. Fluid Mech. 32, 131-144.

SEP, J. 2008 Journal bearing with an intensive axial oil flow-experimental investigation. Scientific Problems of Machines Operation and Maintenance 43, 21-29.

Swift, J. B., Babcock, K. L. \& Hohenberg, P. C. 1994 Effects of thermal noise in TaylorCouette flow with corotation and axial through-flow. Physica A 204, 625-649.

TAkeuchi, D. I. \& Jankowski, D. F. 1981 Numerical and experimental investigation of the stability of spiral Poiseuille flow. J. Fluid Mech. 102, 101-26.

TAYLOR, G. I. 1923 Stability of a viscous liquid contained between two rotating cylinders. Phil. Trans. R. Soc. Lond. 223, 289-343.

Tsameret, A. \& Steinberg, V. 1991a Convective vs. absolute instability in Couette-Taylor flow with an axial flow. Europhys. Lett. 14, 331-336. 
Tsameret, A. \& Steinberg, V. $1991 b$ Noise-modulated propagating pattern in a convectively unstable system. Phys. Rev. Lett. 67, 3392-3395.

Tsameret, A. \& Steinberg, V. 1994 Absolute and convective instabilities and noise-sustained structures in the Couette-Taylor system with an axial flow. Phys. Rev. E 49, 1291-1308.

Vohr, J. A. 1968 An experimental study of Taylor vortices and turbulence in flow between eccentric rotating cylinders. Trans. ASME: J. Lubric. Tech. 90, 285-296.

Younes, M. A. M. A. 1972 The hydrodynamic stability of spiral flow between eccentric rotating cylinders. PhD thesis, Leeds University.

Younes, M. A. M. A., Mobbs, F. R. \& Coney, J. E. R. 1972 Hydrodynamic stability of the flow between eccentric rotating cylinders with axial flow: torque measurements (paper C76/72). In Tribology convention, Institution of Mechanical Engineers, pp. 14-19. 